\title{
A spectroscopic study of southern (candidate) $\gamma$ Doradus stars ${ }^{\star}$
}

\section{Time series analysis ${ }^{\star}$}

\author{
P. De Cat ${ }^{1,2}$, L. Eyer ${ }^{3}$, J. Cuypers ${ }^{1}$, C. Aerts ${ }^{2,4}$, B. Vandenbussche ${ }^{2}$, K. Uytterhoeven ${ }^{2,5}$, K. Reyniers ${ }^{2}$, \\ K. Kolenberg ${ }^{2,6}$, M. Groenewegen ${ }^{2}$, G. Raskin ${ }^{2,7}$, T. Maas ${ }^{2}$, and S. Jankov ${ }^{8,9}$ \\ 1 Royal Observatory of Belgium, Ringlaan 3, 1180 Brussel, Belgium \\ e-mail: peter@oma.be \\ 2 Instituut voor Sterrenkunde, Katholieke Universiteit Leuven, Celestijnenlaan 200 B, 3001 Leuven, Belgium \\ 3 Observatoire de Genève, 1290 Sauverny, Switzerland \\ 4 Department of Astrophysics, Radboud University Nijmegen, PO Box 9010, 6500 GL Nijmegen, The Netherlands \\ 5 Centre for Astrophysics, University of Central Lancashire, Preston, PR1 2HE, UK \\ ${ }^{6}$ Institut für Astronomie, Universität Wien, Türkenschanzstrasse 17, 1180 Wien, Austria \\ 7 Mercator Telescope, Calle Alvarez de Abreu 70, 38700 Santa Cruz de La Palma, Spain \\ 8 Observatoire de la Côte d'Azur, Département Gemini, UMR 6203, 06304 Nice Cedex 4, France \\ 9 Laboratoire Univ. d'Astroph. de Nice (LUAN), UMR 6525, Parc Valrose, 06108 Nice Cedex 02, France
}

Received 17 June 2005 / Accepted 27 October 2005

\section{ABSTRACT}

We present the results of a spectroscopic study of 37 southern (candidate) $\gamma$ Doradus stars based on échelle spectra. The observed spectra were cross-correlated with the standard template spectrum of an F0-type star for easier detection of binary and intrinsic variations. We identified 15 objects as spectroscopic binaries, including 7 new ones, while another 3 objects are binary suspects. At least 12 objects show composite spectra. We could determine the orbital parameters for 9 binaries, of which 4 turned out to be ellipsoidal variables. For 6 binaries, we estimated the expected time-base of the orbital variations. Clear profile variations were observed for 17 objects, pointing towards stellar pulsation. For 8 of them, we have evidence that the main spectroscopic and photometric periods coincide. Our results, in combination with prior knowledge from the literature, lead to the classification of 10 objects as new bona fide $\gamma$ Doradus stars, 1 object as a new bona fide $\delta$ Scuti star, and 8 objects as constant stars. Finally, we determined the projected rotational velocity by two independent methods. The resulting $v \sin i$ values range from 3 to $135 \mathrm{~km} \mathrm{~s}^{-1}$. For the bona fide $\gamma$ Doradus stars, the majority has $v \sin i$ below $60 \mathrm{~km} \mathrm{~s}^{-1}$.

Key words. stars: variables: general - stars: oscillations - line: profiles

\section{Introduction}

The $\gamma$ Doradus stars ( $\gamma$ Dor stars hereafter) are a group of variable early F-type stars situated along the main-sequence. They were recognized as a separate group of pulsating stars one decade ago (Balona et al. 1994b). Their observed variations with typical periods between 0.3 and 3 days are due to $g$-mode pulsations that carry asteroseismic information about the central stellar regions. Their pulsations are driven by a fluxblocking mechanism at the base of their convective envelope (e.g. Guzik \& Kaye 2000; Löffler 2002; Warner et al. 2003; Dupret et al. 2004). Hope of progress in this field boosted many astronomers to observe $\gamma$ Dor stars to allow further constraints

^ Based on observations collected with the CORALIE spectrograph attached to the Euler Telescope of the Geneva Observatory situated at La Silla (Chile).

$\star \star$ Figures 2, 3, 6, 8-10, 13-18, 21-24 are only available in electronic form at http://www. edpsciences.org on both the observed pulsational characteristics and the observed $\gamma$ Dor instability strip in the HR diagram.

The photometric measurements of the satellite mission HIPPARCOS led to an impressive growth in the number of $\gamma$ Dor candidates: from 11 (Krisciunas \& Handler 1995) to 127 (Handler 2002). The number of bona fide members grew from 6 (Krisciunas \& Handler 1995) over 42 (Handler 2002) to 54 (Henry et al. 2005). The reason for dividing the list into two classes, the bona fide members and the candidates (hereafter "bf $\gamma$ Dor" and "cand $\gamma$ Dor", respectively), arises from the fact that the variability of the candidate stars can eventually be attributed to diverse phenomena: eclipsing binaries, ellipsoidal variations, spots, or $\delta$ Scuti (hereafter " $\delta$ Sct") pulsations (due to aliasing problems). Therefore the confirmation that a star belongs to the $\gamma$ Dor star group often requires extensive observations, preferably including spectroscopic ones.

Since most of the bf $\gamma$ Dor stars are multi-periodic, the observed light curves are generally rather complex. Long-term 
monitoring is needed not only to reveal the frequency spectrum, but also to check the stability of the observed periods/phases and amplitudes. Indeed, an excess of scatter around maximum brightness has already been observed in some bf $\gamma$ Dor stars (Zerbi et al. 1997a,b, 1999; Henry et al. 2001; Fekel \& Henry 2003).

In recent years, large scale photometric and spectroscopic follow-up campaigns have been undertaken by other researchers, mainly for northern cand $\gamma$ Dor stars (e.g. Kaye et al. 1999; Henry et al. 2001; Henry \& Fekel 2002, 2003; Fekel et al. 2003; Mathias et al. 2004; Henry \& Fekel 2005; Henry et al. 2005). In our own series of papers, we report on the results obtained for a sample of 37 southern confirmed and cand $\gamma$ Dor stars with time-series of new spectroscopic data gathered in 1998-2003 with the CORALIE spectrograph attached to the 1.2-m Euler telescope located in La Silla (Chile). Paper I (this one) is devoted to the time series analysis of the data. These results are used for the orbital and variability classification of our targets. In Paper II (Bruntt et al., in preparation), the chemical abundances are determined for the slow rotators within our sample. A complementary photometric study has been realised for a slightly different sample of 37 southern (cand) $\gamma$ Dor stars based on new photometric observations in the Johnson-Cousins $B, V, I_{\mathrm{c}}$ filters obtained in 1999-2000 with the MODULAR photometer attached to the 0.5 -m SAAO telescope located in Sutherland (South-Africa). The first results of this campaign were discussed by Bouckaert (2001) and Eyer et al. (2002), while Eyer et al. (in preparation) are preparing the full report. Furthermore, the 1.2-m Mercator telescope located on the Canary Island of La Palma (a twin of the Euler telescope) has become operational since 2001 and is currently used to monitor, amongst others, northern (cand) $\gamma$ Dor stars photometrically in the Geneva $U, B_{1}, B, B_{2}, V_{1}, V, G$ passbands. The results obtained after 18 months of observations are discussed by De Ridder et al. (2004). The results after 3 years of observation will become available soon (Cuypers et al., in preparation).

This paper is organised as follows. The data-sets obtained with the CORALIE spectrograph, which are described in Sect. 2, are used for three main purposes: (1) to determine which of the objects are binaries, and, if possible, to determine their orbits (Sect. 3), (2) to confirm/reject the classification of bf or cand $\gamma$ Dor stars by studying the intrinsic variability of the spectra (Sect. 4), (3) to determine accurate values for the projected rotational velocity $v \sin i$ for each target star (Sect. 5). In Sect. 6, we end with our general conclusion and future prospects.

\section{Description of the data-sets}

A sample of 37 southern confirmed and cand $\gamma$ Dor stars were selected for spectroscopic monitoring from the lists given by Eyer (1998), Aerts et al. (1998), and Handler (1999). For each object, the HD number, the HIPPARCOS number, the Johnson $V$ magnitude, and the spectral classification as found in the SIMBAD astronomical database are given in Cols. (1)-(4) of Table 1 . The 28 targets that are in common with those selected for photometric monitoring (Eyer et al., in preparation) are indicated by an asterisk.
Between Nov. 1998 and Dec. 2002, we gathered highresolution spectra during 14 observation campaigns (Table 2) with the CORALIE spectrograph attached to the 1.2-m EULER telescope located in La Silla (Chile). CORALIE is a 2-fiber-fed échelle spectrograph ( $2^{\prime \prime}$ fibres on the object and sky, respectively), which covers the $388-681 \mathrm{~nm}$ region in 68 orders with a spectral resolution of 50000 . During each night, several exposures were taken with a tungsten lamp to measure the relative pixel sensitivity variation of the CCD. Exposures with a thorium lamp were taken for the wavelength calibration. The science exposure times were chosen for each object individually to result in an $\mathrm{S} / \mathrm{N}$ ratio of about 100 . The resulting number of science exposures for each target and their total time span expressed in days are given in Cols. (5) and (6) of Table 1, respectively.

Right after taking the science exposure, an optimised pipeline reduction was carried out with the INTER-TACOS software. The wavelength calibrated 2D science spectra were used without either rebinning or merging the orders to calculate normalised cross-correlation functions (CCFs) in the solar system barycentric reference frame in the same way as described by Baranne et al. (1996). For all objects, we took the standard template spectrum of an F0-type star as the correlation mask. Given their $\mathrm{S} / \mathrm{N}$ ratio, the time-series of CCFs allow a much easier detection of (double-lined) binaries (see Sect. 3) and/or correlation profile variations (CPVs; see Sect. 4) compared to the time-series of individual spectral lines.

The CCFs were used to determine the radial velocity $\left(v_{\text {rad }}\right)$. In a first approach, $v_{\text {rad }}$ was calculated by fitting the CCF with a Gaussian distribution function. This approach was used only for double-lined objects in phases where the central parts of the lines of the different components are separated well enough in velocity. In a second approach, the first normalised velocity moment (as defined by Aerts et al. 1992) of the CCF is used to calculate $v_{\text {rad }}$. This approach, which is favourable in the case of pronounced CPVs, was used for all our singlelined objects. The accuracy of the obtained $v_{\text {rad }}$ values remains well below $1 \mathrm{~km} \mathrm{~s}^{-1}$ (cf. Table 4). The $v_{\text {rad }}$ time-series were used to search for orbital/intrinsic periods in the observed variations. To check the consistency between the spectroscopic and photometric variability, we also used the HIPPARCOS $H_{\mathrm{p}}$ measurements (ESA 1997). The main HIPPARCOS period in days is given in Col. (7) of Table 1.

Since the spectroscopic data of both HD 12901 and HD 48501 already have been analysed in detail by Aerts et al. (2004), these objects will not be discussed again in the following sections, although they do belong to the sample.

\section{Orbital variations}

The time-series of $v_{\text {rad }}$ are subjected to a period search for the objects for which we have enough data. Two period search algorithms were used: (1) phase dispersion minimization (PDM hereafter; Stellingwerf 1978), and (2) the Lomb-Scargle periodogram (Scargle hereafter; Scargle 1982).

In a first step, we searched for orbital variations. Before our study, 8 of our targets were classified as confirmed or suspected binaries (indicated with ${ }^{+}$in Col. (8) of Table 1). 
Table 1. Overview of the 37 targets discussed in this paper. For each object, we give: the HD number (1), the HIPPARCOS number (2), the Johnson $V$ magnitude (3) and the spectral classification (4) as found in the SIMBAD astronomical database; the number of CORALIE spectra (5) and their total time span expressed in days (6); the main HIPPARCos period in days (7); the orbital classification (8); an indication of the observation of cross-correlation profile variations (9); the variability classification (10); and the mean $v \sin i$ values obtained by least-square fitting with synthetic profiles for the primary (11) and secondary (12) components. The errors are given between brackets in units of the last decimal. The targets that are in common with those of the Eyer et al. sample (in preparation) are indicated by $*$ in Col. (1). The stars that were already classified as confirmed or suspected binaries are indicated by ${ }^{+}$in Col. (8). The stars already classified as bf $\gamma$ Dor stars are indicated by ${ }^{\circ}$ in Col. (10).

\begin{tabular}{|c|c|c|c|c|c|c|c|c|c|c|c|c|}
\hline \multirow{2}{*}{$\begin{array}{l}\text { HD } \\
\text { (1) }\end{array}$} & \multirow{2}{*}{$\begin{array}{l}\text { HIP } \\
\\
\text { (2) }\end{array}$} & \multirow{2}{*}{$\begin{array}{c}m_{V} \\
(\mathrm{mag}) \\
(3)\end{array}$} & \multirow{2}{*}{$\begin{array}{l}S p T \\
(4)\end{array}$} & \multirow{2}{*}{$\begin{array}{r}\# \\
(5)\end{array}$} & \multirow{2}{*}{$\begin{array}{c}\Delta T \\
\text { (days) } \\
(6)\end{array}$} & \multirow{2}{*}{$\begin{array}{c}P_{\text {hipp }} \\
\text { (days) } \\
(7)\end{array}$} & & \multirow{2}{*}{$\begin{array}{l}\text { binarity } \\
(8)\end{array}$} & \multirow{2}{*}{$\begin{array}{l}\text { CPVs } \\
\text { (9) }\end{array}$} & \multirow{2}{*}{$\begin{array}{l}\text { variability } \\
\text { (10) }\end{array}$} & \multicolumn{2}{|c|}{$\begin{array}{c}v \sin i \\
\left(\mathrm{~km} \mathrm{~s}^{-1}\right)\end{array}$} \\
\hline & & & & & & & & & & & (11) & $(12)$ \\
\hline 5590 & 4481 & 9.21 & $\mathrm{~F} 2 \mathrm{~V}$ & 16 & 1355 & & & SB2 & & constant & $103(3)$ & $3.4(0.3)$ \\
\hline 7455 & 5745 & 8.47 & G3 V & 19 & 1723 & & & & no & constant & $3.3(3)$ & \\
\hline 8393 & 6387 & 9.49 & $\mathrm{~F} 7 \mathrm{w}$ & 14 & 1136 & & & SB2 & & constant & $4.1(3)$ & $3.7(2)$ \\
\hline $10167 *$ & 7649 & 6.67 & F0 V & 15 & 1721 & & & $\mathrm{SB} 2^{+}$ & & cand $\gamma$ Dor & $4.5(5)$ & $4.9(6)$ \\
\hline $12901 *$ & 9807 & 6.74 & F0 & 50 & 1721 & 2.18: & [2] & & yes & bf $\gamma$ Dor $^{\circ}$ & $64(3)$ & \\
\hline $14940 *$ & 11192 & 6.68 & F0 IV/V & 63 & 685 & 0.50038 & [1] & & yes & bf $\gamma$ Dor & $39(4)$ & \\
\hline 22001 & 16245 & 4.71 & F5 IV/V & 18 & 1719 & & & & no & constant & $12(2)$ & \\
\hline $26298 *$ & 19383 & 8.16 & $\mathrm{~F} 0 / \mathrm{F} 2 \mathrm{~V}$ & 9 & 332 & & & SB1? & & cand $\gamma$ Dor? & $50.9(5)$ & \\
\hline 27290 & 19893 & 4.26 & F4 III & 19 & 1395 & 0.757031 & [1] & & yes & bf $\gamma \operatorname{Dor}^{\circ}(/ \delta$ Sct?) & $55(2)$ & \\
\hline $27377 *$ & 20036 & 7.4 & F0 V & 3 & 13 & 2.8482 & [3] & SB2 & & cand $\gamma$ Dor? & $5.5(4)$ & $5.2(6)$ \\
\hline 27604 & 20109 & 6.08 & F7 IV/V & 3 & 6 & & & VB? & & constant & $67.5(4)$ & $4.3(3)$ \\
\hline 33262 & 23693 & 4.71 & F7 V & 19 & 1722 & 0.28603 & [3] & & no & constant & 10.1(10) & \\
\hline $34025 *$ & 24215 & 7.87 & F2 IV & 8 & 302 & 0.62221 & [1] & SB2 & yes & bf $\gamma$ Dor & $62.8(11)$ & $39(2)$ \\
\hline $35416 *$ & 25183 & 7.53 & F3 V & 11 & 625 & & & SB2 & & cand $\gamma$ Dor? & $6.6(?)$ & $6.0(?)$ \\
\hline $40745 *$ & 28434 & 6.21 & F2 IV & 8 & 334 & 0.82415 & [1] & & yes & bf $\gamma$ Dor & $39(2)$ & \\
\hline $41448 *$ & 28778 & 7.60 & A9 V & 12 & 10 & 0.419912 & [1] & & yes & bf $\gamma$ Dor & $106(3)$ & \\
\hline $48501 *$ & 32144 & 6.26 & F2 V & 34 & 1493 & & & & yes & bf $\gamma$ Dor $^{\circ}$ & $44(4)$ & \\
\hline $65526 *$ & 39017 & 6.98 & A3 & 2 & 1 & 1.28798 & [1] & & yes & bf $\gamma$ Dor $^{\circ}$ & $56(4)$ & \\
\hline $81421 *$ & 46223 & 7.01 & A3 & 63 & 384 & 0.489724 & [1] & $\mathrm{SB}^{+}{ }^{+}$ & & constant & $62.0(4)$ & $42(4)$ \\
\hline $85964 *$ & 48580 & 7.52 & F3 IV/V & 11 & 381 & 0.62425 & [1] & SB1 & & constant & $65(2)$ & \\
\hline 110379 & & 3.65 & F0 V & 8 & 15 & & & & & cand $\gamma$ Dor & $23.9(6)$ & \\
\hline 110606* & 62105 & 7.8 & $\mathrm{~F} 2 \mathrm{~V}$ & 7 & 75 & 0.977 & [2] & $\mathrm{SB}^{+}{ }^{+}$ & & cand $\gamma$ Dor & $5.8(4)$ & $10(2)$ \\
\hline $111709 *$ & 62774 & 9.27 & A3:m... & 5 & 78 & 1.18567 & [1] & SB2 & yes & cand $\gamma$ Dor/CP star? & $61(1)$ & $25(4)$ \\
\hline $111829 *$ & & 9.49 & A1 IV/V & 2 & 0 & & & SB2? & & cand $\gamma$ Dor? & $47(2)$ & $?$ \\
\hline $112685 *$ & 63372 & 7.85 & F0 V & 24 & 385 & 0.61645 & [1] & & yes & bf $\gamma$ Dor & $70(3)$ & \\
\hline $112934 *$ & 63491 & 6.6 & A9 V & 5 & 79 & $0.8:$ & [2] & & & cand $\gamma$ Dor & $70.5(7)$ & \\
\hline $125081 *$ & 69848 & 7.35 & F2 II & 7 & 77 & 0.153981 & [1] & & yes & bf $\delta \mathrm{Sct}$ & $5.6(5)$ & \\
\hline 126516* & 70566 & 8.2 & F3 V & 9 & 76 & 0.493 & [2] & $\mathrm{SB} 1^{+}$ & & cand $\gamma$ Dor & $3.8(3)$ & \\
\hline $135825 *$ & 74825 & 7.31 & F0 & 17 & 450 & 0.76053 & [1] & & yes & bf $\gamma$ Dor & $38(5)$ & \\
\hline $147787 *$ & 80645 & 5.28 & F4 IV & 10 & 377 & 1.45557 & [1] & $\mathrm{SB}^{+}{ }^{+}$ & & cand $\gamma$ Dor & $7.6(?)$ & $25(?)$ \\
\hline $149989 *$ & 81650 & 6.30 & A9 V & 10 & 379 & 0.42658 & [1] & & yes & bf $\gamma$ Dor & $134(3)$ & \\
\hline $167858 *$ & 89601 & 6.62 & F2 V & 7 & 3 & 1.30700 & [1] & $\mathrm{SB} 1^{+}$ & & bf $\gamma$ Dor $^{\circ}$ & $5.0(2)$ & \\
\hline $187028 *$ & 97590 & 7.60 & F0 V & 21 & 179 & 0.69532 & [1] & & yes & bf $\gamma$ Dor & $85(3)$ & \\
\hline $209295 *$ & 108976 & 7.5 & A9/F0 V & 61 & 1399 & 0.885274 & [1] & $\mathrm{SB} 1^{+}$ & yes & bf $\gamma$ Dor $/ \delta \mathrm{Sct}^{\circ}$ & $86(3)$ & \\
\hline 214291 & 111718 & 6.54 & F7 V & 5 & 1329 & 0.87125 & [1] & $\mathrm{SB} 2^{+}$ & & cand $\gamma$ Dor? & $67.6(8)$ & $64.7(7)$ \\
\hline $216910 *$ & 113402 & 6.70 & F2 IV & 11 & 1718 & 0.69349 & [1] & & yes & bf $\gamma$ Dor & $92(3)$ & \\
\hline $218225 *$ & 114127 & 8.72 & F3 IV & 14 & 1327 & 0.86679 & [1] & & yes & bf $\gamma$ Dor & $59(3)$ & \\
\hline
\end{tabular}

[1] ESA (1997); [2] Handler (1999); [3] Koen \& Eyer (2002).

For HD 147787, HD 167858, and HD 209295 (indicated with * in Table 3), an orbital solution was already available. For all targets showing large Doppler shifts (typically $>10 \mathrm{~km} \mathrm{~s}^{-1}$ ) and/or two components in the time series of CCFs, we tried to 
Table 2. Logbook of the spectroscopic observations. The column \# denotes the number of CORALIE spectra taken for the project of southern (cand) $\gamma$ Dor stars during each of the 14 observation runs.

\begin{tabular}{lcl}
\hline \hline Observation run & $\#$ & Observer \\
\hline $26 / 11 / 1998-06 / 12 / 1998$ & 40 & B. Vandenbussche \\
$06 / 04 / 1999-15 / 04 / 1999$ & 42 & K. Kolenberg \\
$28 / 05 / 1999-04 / 06 / 1999$ & 69 & L. Eyer \\
$15 / 10 / 1999-27 / 10 / 1999$ & 108 & B. Vandenbussche \\
$23 / 11 / 1999-06 / 12 / 1999$ & 27 & B. Vandenbussche \\
$16 / 02 / 2000-29 / 02 / 2000$ & 50 & K. Uytterhoeven \\
$17 / 04 / 2000-25 / 04 / 2000$ & 50 & K. Uytterhoeven \\
$20 / 06 / 2000-06 / 07 / 2000$ & 55 & T. Reyniers \\
$03 / 08 / 2000-16 / 08 / 2000$ & 73 & B. Vandenbussche \\
$28 / 09 / 2000-11 / 10 / 2000$ & 74 & K. Uytterhoeven \\
$13 / 11 / 2001-26 / 11 / 2001$ & 3 & G. Raskin \\
$12 / 07 / 2002-25 / 07 / 2002$ & 8 & B. Vandenbussche \\
$21 / 10 / 2002-03 / 11 / 2002$ & 2 & T. Maas \\
$18 / 12 / 2002-29 / 12 / 2002$ & 24 & M. Groenewegen \\
\hline $26 / 11 / 1998-29 / 12 / 2002$ & 625 & \\
\hline
\end{tabular}

fit the large $v_{\text {rad }}$ variations with an orbit by using an altered version of the FORTRAN code VCURVE (Bertiau \& Grobben 1969).

\subsection{Solved binaries}

\subsubsection{Ellipsoidal variables}

We took $2 P_{\text {hipp }}$ (Col. (7) of Table 1) as a first trial for the orbital period with $P_{\text {hipp }}$ the main photometric period as observed in the HIPPARCOS variations. For the double-lined objects HD 34025 (Fig. 1), HD 81421 (Fig. 2), HD 214291 (Fig. 3), and for the single-lined object HD 85964 (Fig. 4), $2 P_{\text {hipp }}$ fits the observed $v_{\text {rad }}$ variations. We therefore classify them as ellipsoidal variables. According to the statistical test of Lucy \& Sweeney (1971), their short-period orbits are all circular. To determine the final orbital elements (Table 3), the orbital period was fixed except for HD 214291, for which the total time span of the CORALIE data is almost 3 times larger than the total time span of the HIPPARCOS data.

Of these, HD 81421 was the only ellipsoidal variable in our sample that was already classified as such (Handler \& Shobbrook 2002). Eyer et al. (2002) classified both HD 81421 and HD 85964 as eclipsing binaries because they observed a difference in the depth of successive minima in the $B, V, I_{\mathrm{c}}$ light curves, while Duerbeck (1997) concluded from the period-colour-luminosity calibration of the HIPPARCOS data that HD 81421 is a contact binary.

Both HD 34025 and HD 214291 were previously classified as multi-periodic $\gamma$ Dor stars by Eyer et al. (2002) and Aerts et al. (1998), respectively. Since CPVs are observed for the primary component of HD 34025 (right panel of Fig. 1), we classify it as a bf $\gamma$ Dor star. In the end, HD 81421 is the only ellipsoidal variable for which we have enough data to search for additional intrinsic variations. However, no CPVs are visible for either of its components, and there is no evidence of intrinsic periods in the residual $v_{\text {rad }}$ data. Hence, HD 81421 should be omitted from the list of cand $\gamma$ Dor stars.

\subsubsection{Non-ellipsoidal variables}

For 2 double-lined binaries, i.e. HD 10167 (Fig. 5) and HD 147787 (Fig. 6), and for 3 single-lined objects, i.e. HD 126516 (Fig. 7), HD 167858 (Fig. 8), HD 209295 (Fig. 9), $P_{\text {hipp }}$ is clearly not due to ellipsoidal variations, but we were still able to determine the orbit (Table 3 ).

Grenier et al. (1999) and Eyer \& Aerts (2000) already classified HD 10167 as a double-lined object. Handler \& Shobbrook (2002) found only very small variations during individual nights in their photometric observations, but night-tonight variations of a few hundredths of a magnitude. With our $15 v_{\text {rad }}$ values, we could determine the orbit for the first time (Table 3, Col. 2). There is no clear detection of CPVs in either of the components. The object HD 147787 consists of two visual components $\left(V_{A}=5.29 \mathrm{mag} ; V_{B}=9.12 \mathrm{mag}\right)$ with a separation of 24.7 mas (Landolt 1969). It is also known as a singlelined spectroscopic binary with an eccentric orbit of $37.4280 \mathrm{~d}$ (Jones 1928). Here we used the $v_{\text {rad }}$ data of both components to determine orbits for a grid of fixed eccentricity values of which we retained the one with the lowest RMS value (Table 3, Col. 7). Given the large errors on the results, this orbit should be fine-tuned with additional spectroscopic observations. To determine the orbits of HD 126515 and HD 167858, we included the $v_{\text {rad }}$ observations given by Fekel et al. (2003) and Fekel \& Henry (2003), respectively. It is the first time that an orbital solution is given for HD 126516 (Table 3, Col. 6). With our $7 v_{\text {rad }}$ observations of HD 167858, we can improve the orbit given by Fekel \& Henry (2003) slightly (cf. their Table 2). For this object, several observations in speckle interferometry are available, but no evidence of a companion has been found so far (McAlister et al. 1987; Mason et al. 1999). Grenier et al. (1999) first considered HD 209295 as a candidate spectroscopic binary. The orbit given in Col. 9 of Table 3 is based on our 61 $v_{\text {rad }}$ values. Our orbit is close to the orbit given by Handler et al. (2002) (see their Table 7), who already used a subset of our data amongst other spectroscopic observations for this purpose.

The objects HD 147787, HD 167858, and HD 209295 are known as multi-periodic variables (Aerts et al. 1998; Handler \& Shobbrook 2002; Fekel \& Henry 2003; Handler 1999; Koen 2001; Handler et al. 2002, and/or Eyer et al. 2002). For HD 167858, Mathias et al. (2004) already observed evident but small line profile variations from their 11 AURÉLIE spectra, but a combined fit of photometric and spectroscopic data was not possible. For HD 10167, no period is found in the HIPPARCOS photometry but it was listed as a mono-periodic $\gamma$ Dor star by Eyer et al. (2002). Of the non-ellipsoidal binaries, HD 209295 is the only one for which we have enough data to search for intrinsic variations in the residual $v_{\text {rad }}$ data (see Sect. 4). We classify the other ones as binaries with a (cand) $\gamma$ Dor component. Because the further exploitation of their dynamical information with, e.g., interferometry can give additional and independent constraints on physical properties of the components, these 
Table 3. Overview of the orbital elements determined for the ellipsoidal variables (HD 34025, HD 81421, HD 214291, HD 85964) and the binaries with a (cand) $\gamma$ Dor component (HD 10167, HD 147787, HD 126516, HD 167858, HD 209295) in our sample of 37 targets. The errors are given between brackets in units of the last decimal. The values that were fixed are given in italics. The targets for which an orbit was already known in the literature are indicated with $*$.

\begin{tabular}{|c|c|c|c|c|c|c|c|c|c|}
\hline & HD 10167 & $\begin{array}{l}\text { HD } 34025 \\
\text { (ellipsoidal) }\end{array}$ & $\begin{array}{c}\text { HD } 81421 \\
\text { (ellipsoidal) }\end{array}$ & $\begin{array}{c}\text { HD } 85964 \\
\text { (ellipsoidal) }\end{array}$ & HD 126516 & $\begin{array}{c}\text { HD } 147787 \\
*\end{array}$ & $\begin{array}{c}\text { HD } 167858 \\
*\end{array}$ & $\begin{array}{c}\text { HD } 209295 \\
*\end{array}$ & $\begin{array}{c}\text { HD } 214291 \\
\quad \text { (ellipsoidal) }\end{array}$ \\
\hline$P_{\text {orb (days) }}$ & $9.3199(2)$ & 1.24442 & 0.97948 & 1.24850 & $2.1245(5)$ & $39.86(8)$ & $4.48510(13)$ & $3.10573(2)$ & $1.74247(2)$ \\
\hline$v_{\gamma}\left(\mathrm{km} \mathrm{s}^{-1}\right)$ & $5.2(2)$ & 28.1(8) & $9.3(4)$ & $4.1(4)$ & $-20.6(13)$ & $-4.9(6)$ & $-28.3(2)$ & $-20.5(2)$ & $-13.9(6)$ \\
\hline$e$ & 0.0 & 0.0 & 0.0 & 0.0 & 0.0 & 0.59 & 0.0 & $0.324(5)$ & 0.0 \\
\hline$t(\tau)(\mathrm{HJD}-2450000)$ & & & & & & $1312(2)$ & & $2865.097(12)$ & \\
\hline$\omega\left({ }^{\circ}\right)$ & & & & & & $97(10)$ & & $33(1)$ & \\
\hline$K_{1}\left(\mathrm{~km} \mathrm{~s}^{-1}\right)$ & $39.7(4)$ & $81(2)$ & $87.8(7)$ & $65.5(5)$ & $33(1)$ & $61(72)$ & $6.3(2)$ & $52.8(3)$ & 111(14) \\
\hline$K_{2}\left(\mathrm{~km} \mathrm{~s}^{-1}\right)$ & $41.8(4)$ & 135(3) & 164.7(7) & & & $67(79)$ & & & 111(14) \\
\hline$a_{1} \sin i(\mathrm{AU})$ & $0.0340(3)$ & $0.0093(2)$ & $0.00790(6)$ & $0.00752(6)$ & $0.0065(3)$ & $0.2(2)$ & $0.00261(8)$ & $0.01426(8)$ & $0.018(2)$ \\
\hline$a_{2} \sin i(\mathrm{AU})$ & $0.0358(3)$ & $0.0154(3)$ & $0.01483(6)$ & & & $0.2(3)$ & & & $0.018(2)$ \\
\hline$M_{1} \sin ^{3} i_{\left(M_{\odot}\right)}$ & $0.268(11)$ & $0.81(9)$ & $1.07(3)$ & & & $2(12)$ & & & $1.0(5)$ \\
\hline$M_{2} \sin ^{3} i\left(M_{\odot}\right)$ & $0.254(8)$ & $0.49(4)$ & $0.568(15)$ & & & $2(9)$ & & & $1.0(4)$ \\
\hline$f(M)\left(M_{\odot}\right)$ & & & & $0.0364(8)$ & $0.0080(9)$ & & $0.00012(2)$ & $0.0401(7)$ & \\
\hline $\mathrm{rms}\left(\mathrm{km} \mathrm{s}^{-1}\right)$ & 0.99 & 1.43 & 2.60 & 0.87 & 1.97 & 0.98 & 0.89 & 1.03 & 0.67 \\
\hline
\end{tabular}

Table 4. The peak-to-peak value $\left(\Delta v_{\text {rad }}\right)$ and the standard deviation $\left(\sigma_{\text {rad }}\right)$ of the $v_{\text {rad }}$ data is given for the objects for which no clear correlation profile variations have been detected in our current set of CORALIE data.

\begin{tabular}{lclll}
\hline \hline HD & HIP & $\Delta v_{\text {rad }}$ & $\sigma_{\text {rad }}$ & \\
\hline 22001 & 16245 & 0.2 & 0.05 & const \\
33262 & 23693 & 0.2 & 0.06 & const \\
7455 & 5745 & 0.3 & 0.07 & const \\
$112934 *$ & 63491 & 1.2 & 0.5 & CPVs? \\
110379 & & 2.5 & 0.8 & CPVs? \\
\hline
\end{tabular}

objects are the most interesting ones in our sample from an asteroseismic point of view.

\subsection{Unsolved binaries}

Finally, there is a group of 9 suspected binaries in our sample consisting of systems for which the amount of data is insufficient to check if we are dealing with an ellipsoidal variable or not and/or to determine an orbital period. All their observed CCFs are shown in Fig. 10. At least 7 of these objects are double-lined.

Eyer \& Aerts (2000) found no intrinsic photometric variations in their GENEVA data for HD 5590 and HD 8393. For HD 35416, which is listed as "microvariable" in the HIPPARCos catalogue (ESA 1997), they found a different result for each period search method. However, their photometric observations of both HD 8393 and HD 35416 show a slight drift. These double-lined objects are clearly long period binaries: their $v_{\text {rad }}$ values barely change within an observation run while clear Doppler shifts are observed between observations of different observation runs. Hence, their orbital period must be much longer than $10 \mathrm{~d}$. Note that if we did not have the first observation of HD 35416 (Fig. 10, upper right panel, lowest
$\mathrm{CCF}$ ), we would have interpreted the CPVs as being due to pulsation instead of binarity.

Of the unsolved binaries, HD 110606 is the only one for which a composite spectrum was observed before. Nordström et al. (1997) determined a mass ratio of 0.912(34). It cannot be an ellipsoidal variable since $2 P_{\text {hipp }}$ does not fit our $v_{\text {rad }}$ data at all. Our orbit determination failed, but the most promising candidate orbital period is about $63 \mathrm{~d}$.

Paunzen \& Maitzen (1998) concluded from the HIPPARCos data that HD 111709 is a new variable chemically peculiar star. The $B, V, I_{\mathrm{c}}$ photometry of HD 27377 shows long-term behaviour that yields a period of about $10 \mathrm{~d}$ (Eyer et al. 2002). However, we suspect that it is a short-period binary since we observed $\Delta v_{\text {rad }} \simeq 3 \mathrm{~km} \mathrm{~s}^{-1}$ in $45 \mathrm{~min}$. Our current spectroscopic data for these double-lined objects are insufficient to confirm or rule out ellipsoidality. Note that CPVs are present in the primary component of HD 111709 (Fig. 10, middle panel), which confirms the $\gamma$ Dor or spotted character of this component.

In the case of HD 27604, HD 26298, and HD 111829 (Fig. 10, bottom panels), it is not clear if we are dealing with binaries or not. Indeed, Eyer \& Aerts (2000) classified HD 27604 as a constant star, but they observed a few data points with a higher-than-normal magnitude, which might indicate the presence of eclipses. It is a close visual binary with a separation of 0.800 arcsec (ESA 1997), which might explain why we observe a double-lined object but without clear Doppler shifts. In the HIPPARCOS catalogue (ESA 1997), HD 26298 is listed as a constant star. Lu et al. (1987) found no evidence of duplicity with speckle interferometry. The GENEVA observations of Eyer \& Aerts (2000) yield periods close to a sampling period, while the period in colour seems to be half the period in $V$, which points towards ellipsoidal variability. We find a peak-to-peak value of $9.5 \mathrm{~km} \mathrm{~s}^{-1}$ in $v_{\text {rad }}$. The CCFs do not show clear asymmetries, but instead global Doppler shifts, which favours an interpretation as a suspected binary. For HD 111829, Mantegazza et al. (1991) observed not very strict periodic light variations 

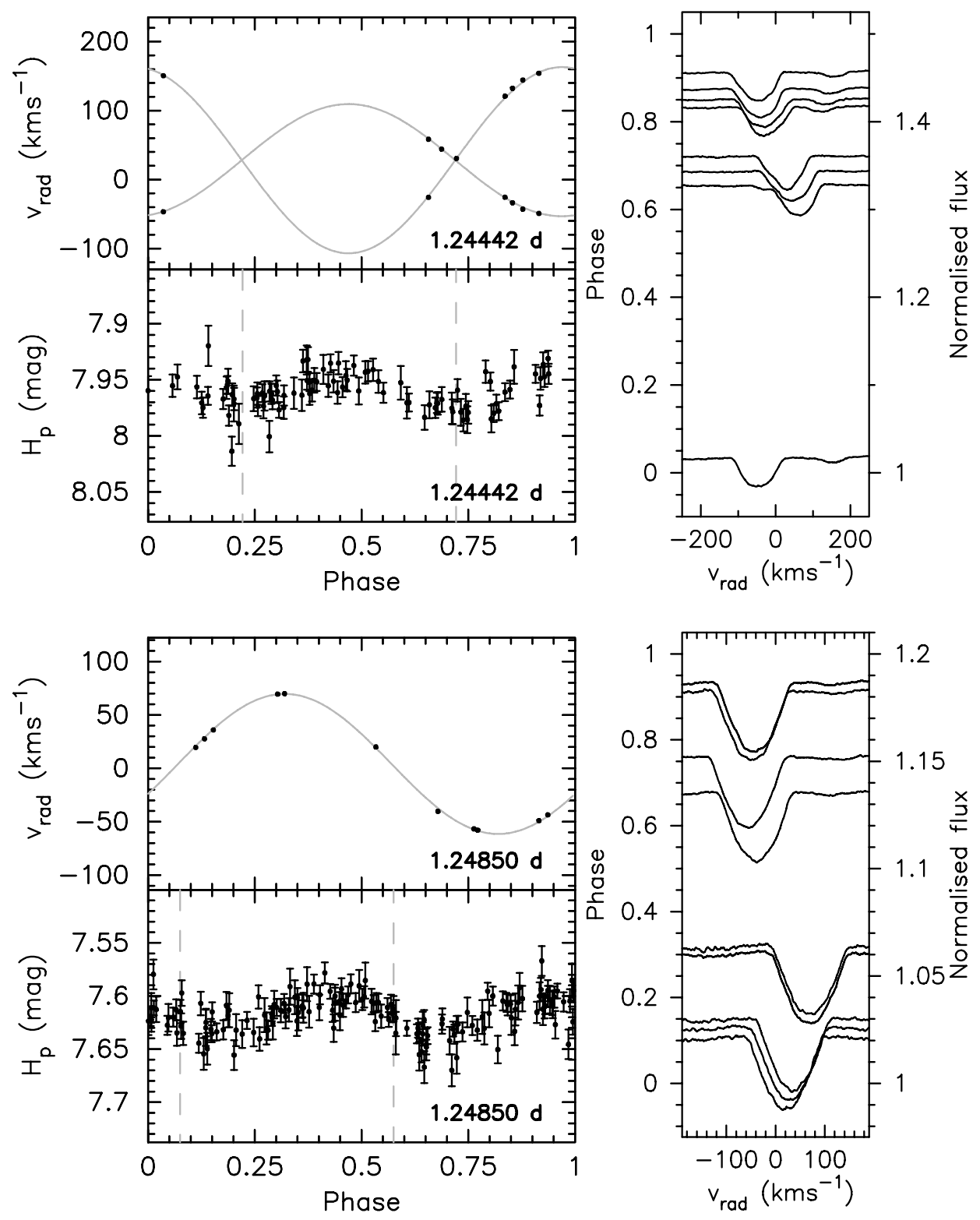

Fig. 1. Phase diagram of the radial velocity $v_{\text {rad }}$ (top left) and the Hipparcos $H_{\mathrm{p}}$ measurements (bottom left) of HD 34025 with the period as given in the bottom right corner. The reference epoch is HJD 2450000. The dashed lines in the bottom left panel denote the phase at which $v_{\text {rad }}=v_{\gamma}$. In the right panel, a selection of observed cross-correlation profiles are shown as a function of orbital phase.
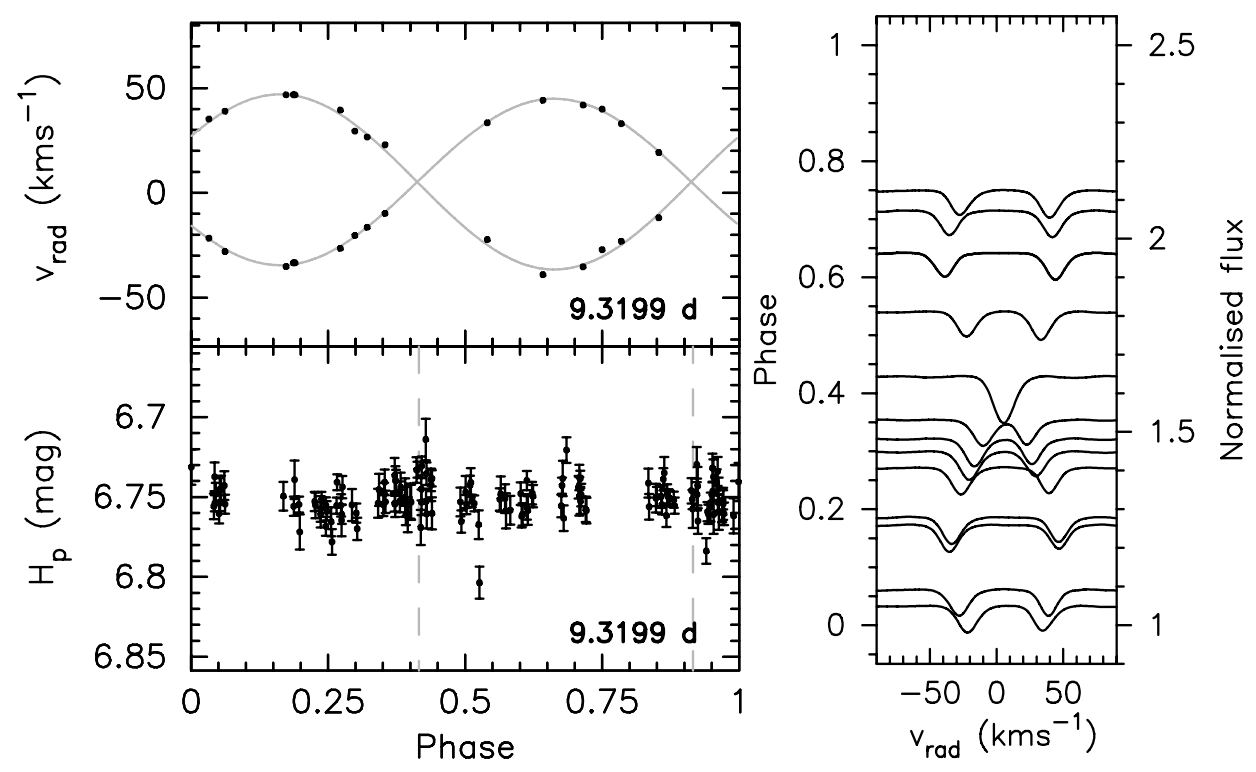

Fig. 4. Same as Fig. 1, but for HD 85964.

Fig. 5. Same as Fig. 1, but for HD 10167. 


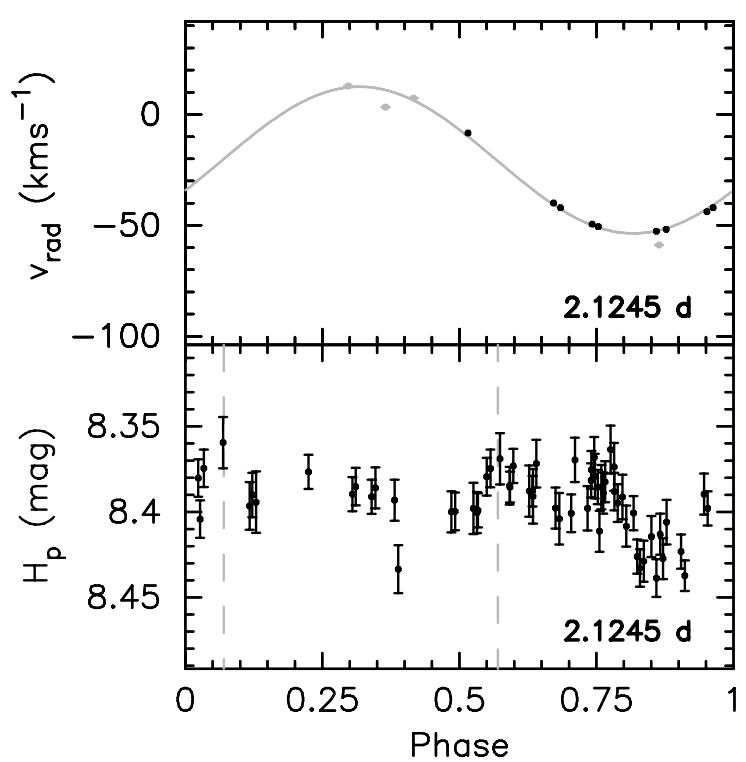

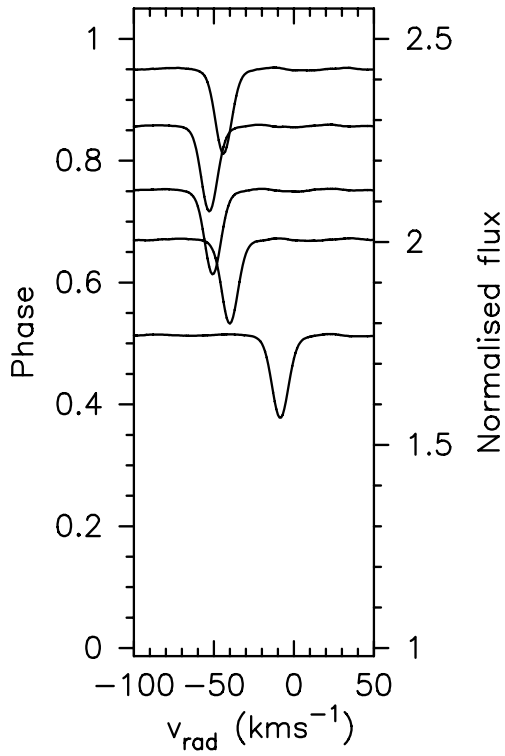

Fig. 7. Same as Fig. 1, but for HD 126516. with a $V$ amplitude of about $0.04 \mathrm{mag}$ on a time-scale of 1.8 or $4 \mathrm{~d}$. Our 2 observations show $\Delta v_{\text {rad }} \simeq 7 \mathrm{~km} \mathrm{~s}^{-1}$ in 1 hour. It is unclear if the obvious line profiles observed in the CCFs are superposed on line profiles of a very rapidly rotating component or not (Fig. 10, lower right panel).

Two of these unsolved binaries were previously classified as multi-periodic variables, i.e. HD 27377 by Handler (1999) and HD 110606 by Eyer et al. (2002). Since we cannot derive their orbits, we are unable to search for intrinsic periods. Moreover, apart from the primary of HD 111709, none of the components of the unsolved binaries shows clear CPVs.

\section{Intrinsic variations}

Before our study, 6 of our targets were classified as bf $\gamma$ Dor stars (indicated by ${ }^{\circ}$ in Col. (10) of Table 1). In the second step, we searched for intrinsic periods in the (residual) $v_{\text {rad }}$ data of all the objects with clear CPVs, as far as the number of data points allowed. In most cases, the resulting periodograms are very noisy. If our data turned out to be insufficient for an independent period search, we made phase diagrams with already known photometric periods to check the possible consistency with the observed spectroscopic variations.

\subsection{Clear correlation profile variations}

\subsubsection{Spectroscopic variations compatible with photometric period}

In our sample, HD 34025, HD 111709 and HD 209295 are the only binaries for which clear CPVs are seen in one of the components (Figs. 1, 10 and 9, respectively). Only for HD 209295 did the number of datapoints and the quality of the derived orbit allow us to search for additional intrinsic periods. Handler et al. (2002) organised a multi-site campaign for this object and detected multi-periodic $\gamma$ Dor and $\delta$ Sct variability. A total of 10 independent periods were found: 7 in photometry $\left(P_{1}^{\mathrm{p}}=0.88529(2) \mathrm{d}, P_{2}^{\mathrm{p}}=0.434363(6) \mathrm{d}, P_{3}^{\mathrm{p}}=0.388230(8) \mathrm{d}\right.$,
$\left.P_{4}^{\mathrm{p}}=0.8519 \mathrm{~d}, P_{5}^{\mathrm{p}}=0.5659 \mathrm{~d}, P_{6}^{\mathrm{p}}=0.4430 \mathrm{~d}, P_{7}^{\mathrm{p}}=0.9246 \mathrm{~d}\right)$, and 3 additional ones in spectroscopy $\left(P_{8}^{\mathrm{s}}=1.03523(12) \mathrm{d}\right.$, $\left.P_{9}^{\mathrm{s}}=0.62113(4) \mathrm{d}, P_{10}^{\mathrm{s}}=0.345075(13) \mathrm{d}\right)$. However, $P_{3}^{\mathrm{p}}, P_{6}^{\mathrm{p}}$, $P_{8}^{\mathrm{s}}, P_{9}^{\mathrm{s}}$, and $P_{10}^{\mathrm{s}}$ are suspected to be tidally excited since they are exact subharmonics of the orbital period. We have evidence that at least 4 of the known periods are also present in the residual $v_{\text {rad }}$ data after prewhitening the orbit given in Table 3: $P_{1}=0.4343(2) \mathrm{d} \simeq P_{2}^{\mathrm{p}}, P_{2}=0.8853(9) \mathrm{d} \simeq P_{1}^{\mathrm{p}}$, $P_{3}=0.8535(9) \mathrm{d} \simeq P_{4}^{\mathrm{p}}$, and $P_{4}=0.3882(2) \mathrm{d} \simeq P_{3}^{\mathrm{p}}$ (Fig. 11). During the period search, $0.8650(9) \mathrm{d}$ originally seemed to be the best candidate for the second period. However, since $(1 / 0.8650) \simeq\left(1 / P_{1}^{\mathrm{p}}+1 / P_{4}^{\mathrm{p}}\right) / 2$, and since prewhitening with $0.8650 \mathrm{c} \mathrm{d}^{-1}$ or with $P_{2}$ and $P_{3}$ reduces the variance with the same amount, we favoured the last two. We refer to Handler et al. (2002) for a more extensive study of this star.

For the objects HD 40745 (Fig. 12), HD 41448 (Fig. 13), HD 112685 (Fig. 14), and HD 187028 (Fig. 15), a period close to $P_{\text {hipp }}$ (or one of its aliases) is found as one of the best fitting periods from an independent search in our $v_{\text {rad }}$ data. We therefore have evidence that the main photometric and spectroscopic periods coincide for these objects. These periods are not connected to binarity, and since the $v_{\text {rad }}$ variations are sinusoidal, they are probably not due to spots. Moreover, HD 40745, HD 41448, and HD 187028 show multi-periodic variations in their HIPPARCOS observations (e.g. Handler 1999), while HD 112685 was found to be multi-periodic in $B, V, I_{\mathrm{c}}$ observations (Eyer et al. 2002). We can therefore classify them as bf $\gamma$ Dor stars. Note that Mathias et al. (2004) failed to observe line profile variations in their AURÉLIE data of HD 40745 and HD 41448. In the case of HD 40745, we cannot confirm any of the alternative HIPPARCOS periods given by Aerts et al. (1998).

The period $P_{\text {hipp }}=0.15398 \mathrm{~d}$ observed in the HIPPARCOS observations of HD 125081 (ESA 1997) is typical of $\delta$ Sct stars. Paunzen \& Maitzen (1998) classified this object as a new variable, chemically peculiar star (F3 SrCrEu). Our observations confirm the $\delta$ Sct character of HD 125081, since $P_{\text {hipp }}$ is clearly the main period in spectroscopy (Fig. 16). 

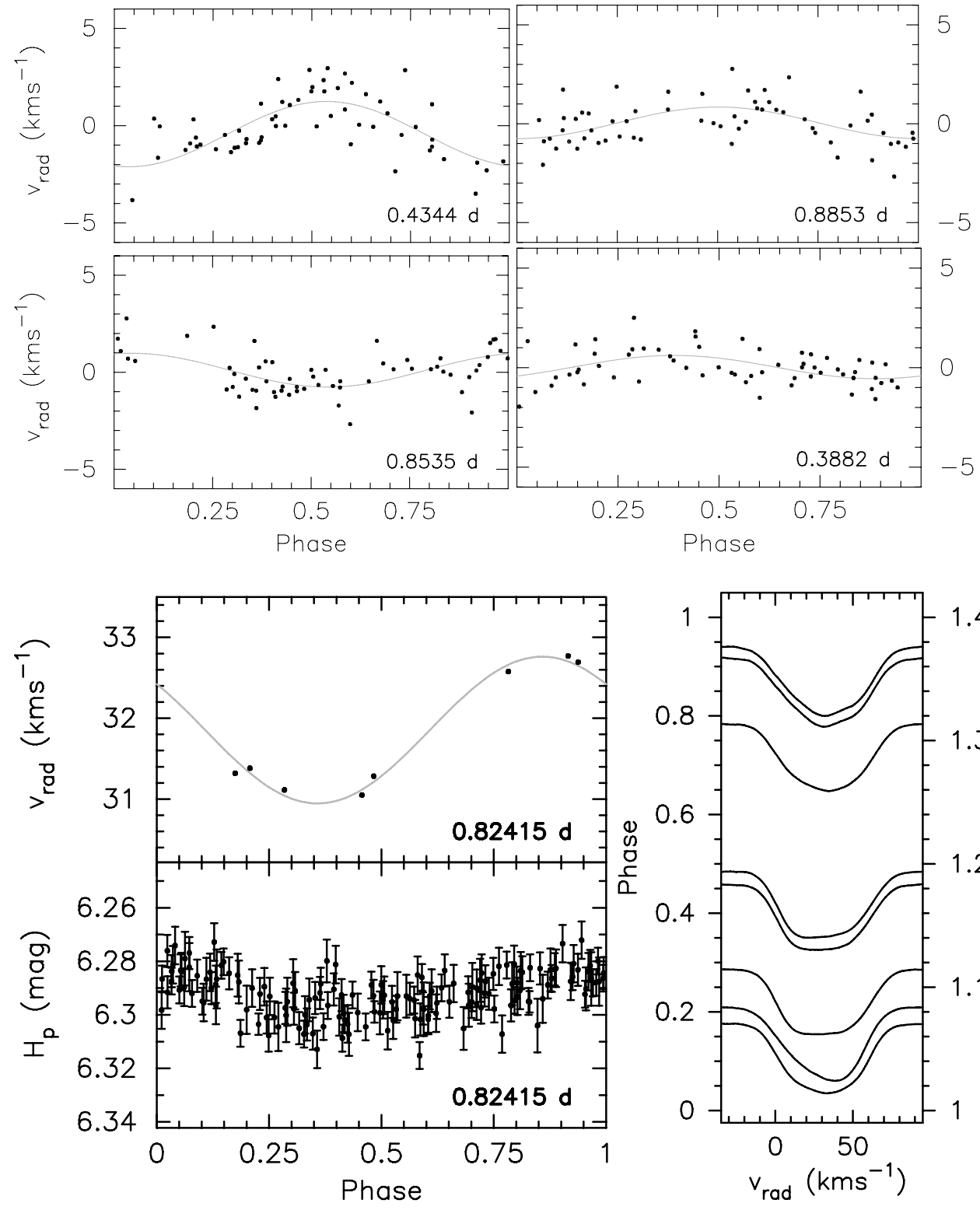

For HD 135825 and HD 218225, none of the highest peaks in the periodograms correspond to a period close to $P_{\text {hipp }}$ or one of its aliases. The best fitting periods, 0.63(3) d and 2.0210(4) d respectively, reduce the variance with more than $80 \%$. However, for both objects, low peaks do occur near $P_{\text {hipp }}$ in the periodograms, and these periods already reduce the variance with $\sim 60 \%$. Hence, given the small number of datapoints, we cannot rule out that $P_{\text {hipp }}$ coincides with the main spectroscopic period. Phase diagrams with $P_{\text {hipp }}$ are given in the left panels of Figs. 17 and 18. Since both HD 135825 and HD 218225 are multi-periodic in photometry (Eyer et al. 2002; Aerts et al. 1998), we now classify them as bf $\gamma$ Dor stars.

\subsubsection{Spectroscopic variations not compatible with photometric period}

For HD 14940, two periods were detected in the HIPPARCos data: $0.5004 \mathrm{~d}$ and $0.9800 \mathrm{~d}$ (Aerts et al. 1998). However, they out this result.
Fig. 11. Phase diagrams for the intrinsic periods of HD 209295 found in the residual $v_{\text {rad }}$ data after prewhitening with the orbit given in Table 3. The reference epoch is HJD 2450000. Top left: $P_{1}=0.4343(2)$ d. Top right: $P_{2}=0.8853(9)$ d (prewhitened with $\left.P_{1}\right)$. Bottom left: $P_{3}=$ 0.8535(9) d (prewhitened with $P_{1}$ ). Bottom right: $P_{4}=0.3882(2) \mathrm{d}$ (prewhitened with $P_{1}, P_{2}$ and $P_{3}$ ).

Fig. 12. Phase diagram of the radial velocity $v_{\text {rad }}$ (top left) and the Hipparcos $H_{\mathrm{p}}$ measurements (bottom left) of HD 40745 with the period as given in the bottom right corner. The reference epoch is HJD 2450000. In the right panel, a selection of observed cross-correlation profiles are shown as a function of pulsation phase.

do not fit our $v_{\text {rad }}$ data at all. These periods reduce the variance with less than $10 \%$, while the best fitting period, $0.3946 \mathrm{~d}$, induces a variance reduction of more than $50 \%$ (Fig. 19). After prewhitening, there is still power near $0.5842 \mathrm{~d}, 0.9647 \mathrm{~d}$, and $0.4234 \mathrm{~d}$. We are clearly dealing with a multi-periodic $\gamma$ Dor star, but the aliasing is too strong to continue our period search.

For $\gamma$ Dor (=HD 27290), three periods are commonly present in photometry and spectroscopy: $0.75701 \mathrm{~d}, 0.73339 \mathrm{~d}$, and $0.67797 \mathrm{~d}$ (Balona et al. 1994a,b, 1996). However, we find a $\delta$ Sct like period instead: $0.1103 \mathrm{~d}$ reduces the variance with $90 \%$ while the variance reduction for the known periods are all below $45 \%$ (Fig. 20). We do stress that the periodograms are very noisy, and we clearly need more data to confirm or rule

Aerts et al. (1998) found two periods in the HIPPARcos data of both HD 149989 and HD 216910. In the case of HD 216910, none of these periods could be confirmed with the $B, V, I_{\mathrm{c}}$ observations by Eyer et al. (2002). Our current 

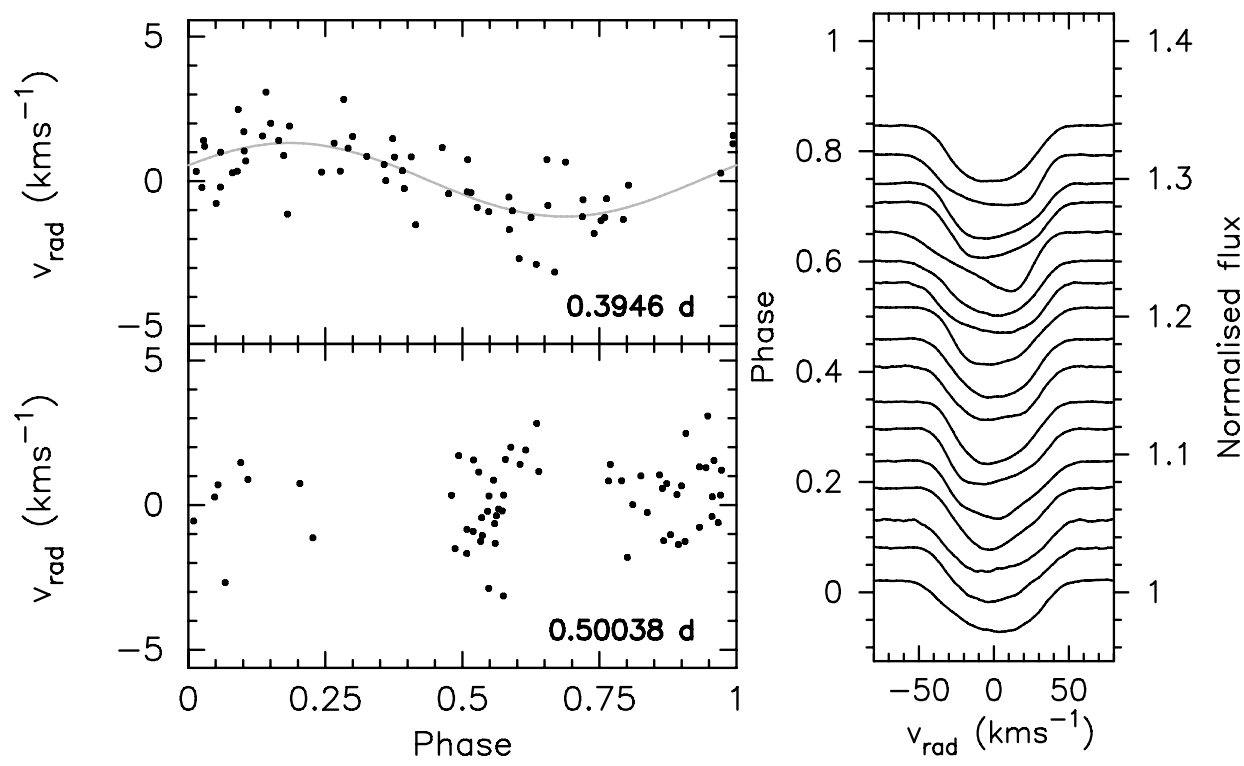

Fig. 19. Phase diagram of the radial velocity $v_{\text {rad }}$ with our best fitting period (top left) and the best fitting photometric period (bottom left) of HD 14940. The period values are given in the bottom right corner. The reference epoch is HJD 2450000 . In the right panel, a selection of observed cross-correlation profiles are shown as a function of our best fitting period.
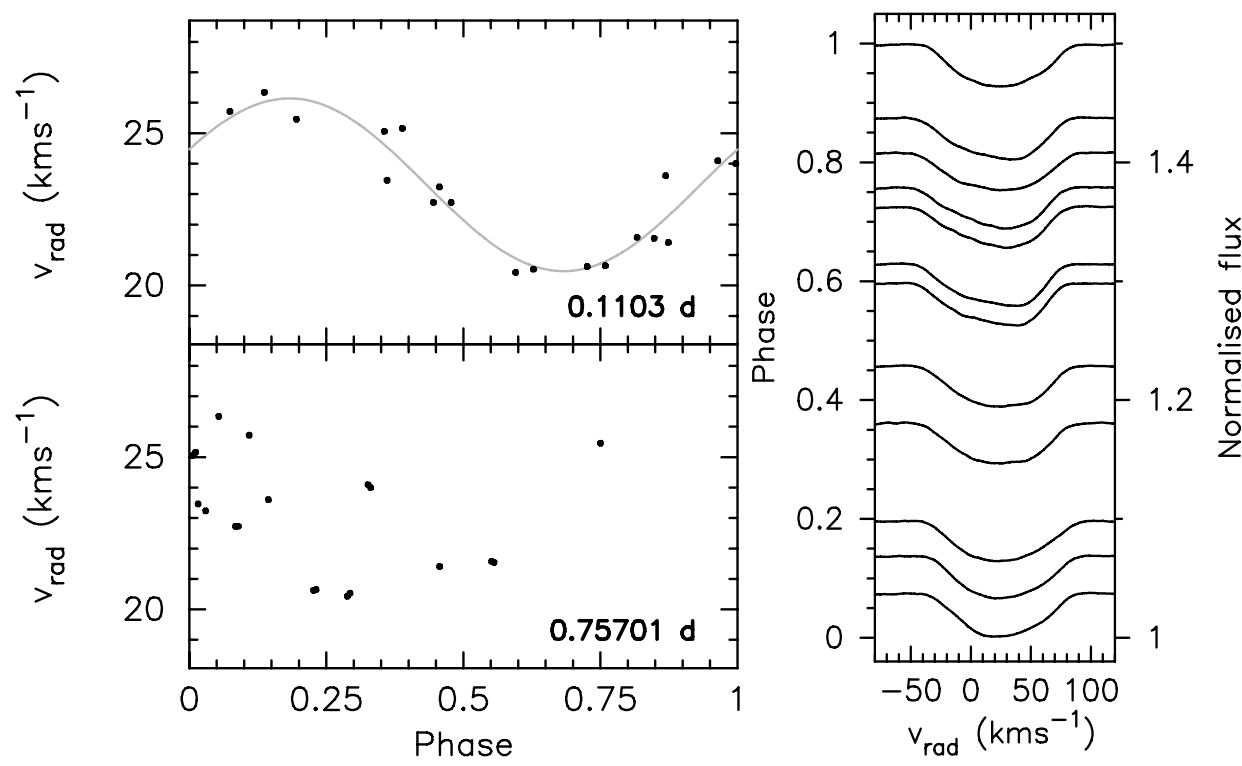

Fig. 20. Same as Fig. 19, but for HD 27290.

amount of CORALIE data is insufficient for an independent period search. However, phase plots with the HIPPARCOS periods enable us to rule out their presence in our spectroscopic observations (Figs. 21 and 22). Due to the broad CCPs, the CPVs of HD 149989 are not as clear as for the objects discussed in Sect. 4.1 so far.

\subsubsection{Unsolved intrinsic variations}

For HD 65526, the HIPPARCos team found $P_{\text {hipp }}=$ 1.28798 days as the main period (ESA 1997). A total of four other periods were derived with the HIPPARCOS data by Handler (1999) and by Martín et al. (2003), who confirmed one of them in their Strömgren data. Mathias et al. (2004) did not detect asymmetries in their 1 AURÉLIE spectrum, while the CPVs are obvious in our 2 CCFs (Fig. 23).

\subsection{No clear correlation profile variations}

There are five (apparently) single objects for which no clear CPVs are detected in our CORALIE data (Fig. 24). Both HD 7455 and HD 22001 were found to be constant in GENEVA photometry (Eyer \& Aerts 2000). For HD 33262, a period of $3.4961 \mathrm{~d}$ was found in the HIPPARCos data (Koen \& Eyer 2002), while only some indications of a period of about 100 days were found in the GENEVA data (Eyer \& Aerts 2000). For these three objects, we found peak-to-peak values $\Delta v_{\text {rad }}$ below $1 \mathrm{~km} \mathrm{~s}^{-1}$ and corresponding standard deviations $\sigma_{\text {rad }}$ well below $0.1 \mathrm{~km} \mathrm{~s}^{-1}$ (Table 4 ), so we classify them as spectroscopically constant.

In the HIPPARCos data of HD 112934, Handler (1999) found evidence of a period of $\sim 0.8$ days. Eyer et al. (2002) confirmed its classification as a mono-periodic $\gamma$ Dor star on the basis of new $B, V, I_{\mathrm{c}}$ observations. For HD 110379 , no HIPPARCOS observations are available, but Krisciunas \& Handler (1995) observed a period of 0.228 days in Strömgren 


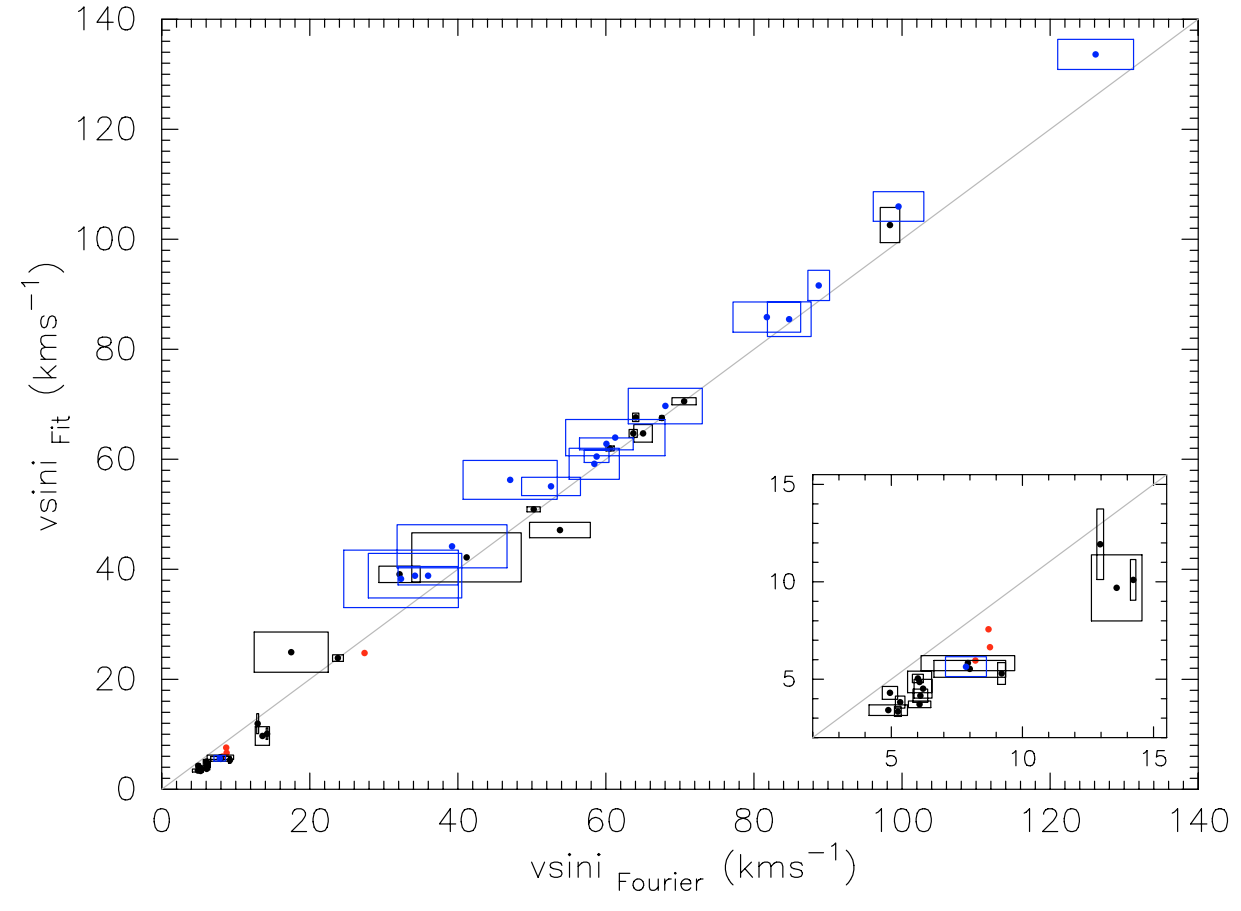

Fig. 25. Comparison of the $v \sin i$ values (full circles with error boxes) determined with the Fourier method ( $\left.v \sin i_{\text {Fourier }}\right)$ to those determined by a least-square fitting with synthetic profiles $\left(v \sin i_{\text {Fit }}\right)$. The components known to be pulsating are given in blue, and those for which the $v \sin i$ value is only based on 1 crosscorrelation profile are given in red. The colour representation is only available in the online version of the paper. photometry. With our CORALIE data alone, it is not clear if we are dealing with pulsating stars or not. No clear CPVs were observed (Fig. 24), but their $\sigma_{\text {rad }}$ values are at least 10 times larger than the one of HD 22001.

\section{Projected rotational velocity}

The CCFs were used to determine the projected rotational velocity $(v \sin i)$. For the double-lined objects, only the CCFs in which the profiles of both components are well separated are used (if available). The $v \sin i$ values were determined with the Fourier method ( $v \sin i_{\text {Fourier }}$; Gray 1992) and by a least-square fitting with rotationally broadened synthetic profiles with a Gaussian intrinsic width but without pulsational broadening ( $\left.v \sin i_{\text {Fit }}\right)$. For each object, a value of 0.555 was taken for the limb-darkening coefficient.

In Fig. 25, the resulting $v \sin i$ values and their corresponding standard deviations, as found with both methods, are compared for each component. For $v \sin i>15 \mathrm{~km} \mathrm{~s}^{-1}$, the results of both methods are generally fully compatible, while for $v \sin i<15 \mathrm{~km} \mathrm{~s}^{-1}$ (see enlargement in Fig. 25), we systematically found $v \sin i_{\text {Fourier }}>v \sin i_{\text {Fit }}$. For slow rotators, there is confusion in the zeros of the Fourier transform invoked by other broadening mechanisms than rotation. Moreover, the standard deviations on the $v \sin i$ values found with the Fourier method are in general larger than those found with least-square fitting. We therefore favour the results of the least-square fitting and list the $v \sin i_{\text {Fit }}$ values for the primary and secondary components in Cols. (11) and (12) of Table 1, respectively. Although the cross-correlation profiles of pulsating components show asymmetries, we do not detect significant differences in the relative errors on $v \sin i$ of the pulsating and non-pulsating

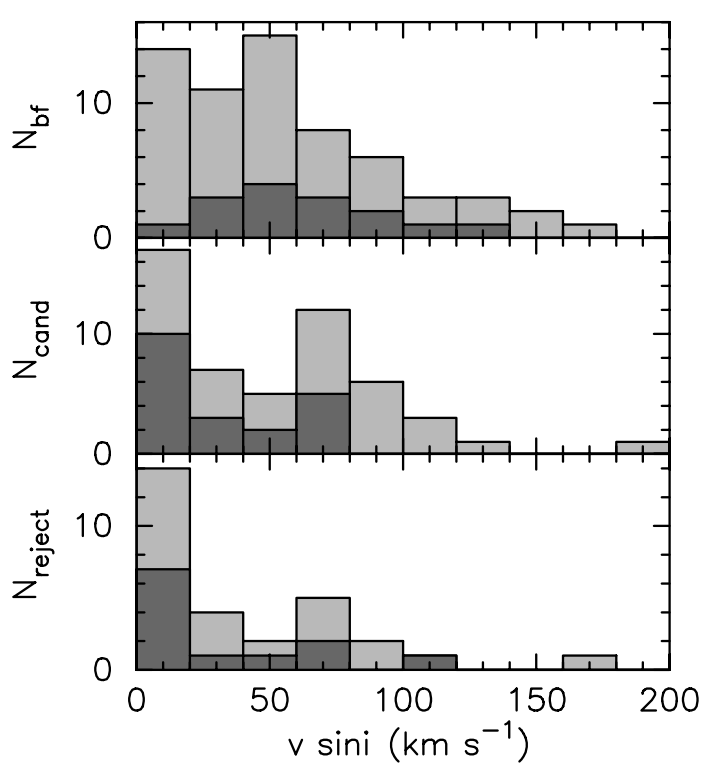

Fig. 26. Histograms showing the number of bf $\gamma$ Dor stars $\left(N_{\mathrm{bf}}\right.$; top $)$, cand $\gamma$ Dor stars $\left(N_{\text {cand }} ;\right.$ middle $)$, and rejected $\gamma$ Dor stars that were formerly under consideration $\left(N_{\text {reject }} ;\right.$ bottom $)$ as a function of $v \sin i$. The $v \sin i$ values from this work are given in dark grey. The other values were taken from Handler (2002); Henry \& Fekel (2002, 2003); Fekel et al. (2003); Mathias et al. (2004); Henry \& Fekel (2005); Henry et al. (2005).

components in our sample, given in blue and black, respectively, in Fig. $25^{1}$.

In Fig. 26, we show histograms of the number of bf $\gamma$ Dor stars $\left(N_{\mathrm{bf}}\right)$, cand $\gamma$ Dor stars $\left(N_{\text {cand }}\right)$, and rejected $\gamma$ Dor stars (i.e. objects that were formerly under consideration; $N_{\text {reject }}$ ), as a function of $v \sin i$. As input for the different groups, we used a compilation of our results and those listed by

1 The colours are given in the electronic version only. 
Table 5. An overview of the orbital classification, i.e. apparently single star ("single"), suspected binary ("suspect"), single-lined spectroscopic binary ("SB1"), and double-lined spectroscopic binary ("SB2"), and variability classification of the objects discussed in this paper. The stars in italics will be the subject of dedicated future studies.

\begin{tabular}{|c|c|c|c|c|c|c|}
\hline & \multicolumn{2}{|c|}{ bf $\gamma$ Dor star } & \multicolumn{2}{|c|}{ cand $\gamma$ Dor star } & \multicolumn{2}{|c|}{ rejected $\gamma$ Dor star } \\
\hline single & 13 & $\begin{array}{l}\text { HD 12901, HD 14940, HD } 27290, \\
\text { HD } 40745, \text { HD } 41448, \text { HD } 48501, \\
\text { HD } 65526, \text { HD } 112685, \text { HD } 135825, \\
\text { HD } 149989, \text { HD } 187025, \text { HD } 216910, \\
\text { HD } 218225\end{array}$ & & HD 110379, HD 112934 & & $\begin{array}{l}\text { HD } 7455, \\
\text { HD } 22001, \\
\text { HD } 33262, \\
\text { HD } 125081^{1}\end{array}$ \\
\hline suspect & 0 & & 2 & HD 111829, HD 26298 & & HD 27604 \\
\hline SB1 & 2 & $H D 167858^{2}, H D 209295$ & 1 & $H D 126516$ & & HD 85964 \\
\hline SB2 & & HD 34025 & 7 & $\begin{array}{l}H D 10167, H D 27377^{3}, H D 35416 \\
H D 110606, H D 111709^{3,4}, H D 147787, \\
H D 214291\end{array}$ & & $\begin{array}{l}\text { HD } 5590, \\
\text { HD } 8393, \\
\text { HD } 81421\end{array}$ \\
\hline
\end{tabular}

${ }^{1}$ bf $\delta$ Sct star; ${ }^{2}$ shows no cross-correlation profile variations but was classified as a bf $\gamma$ Dor star before; ${ }^{3}$ ellipsoidal variability instead of pulsation cannot be ruled out; ${ }^{4}$ shows cross-correlation profile variations, but was classified as a chemically peculiar star before.

Handler (2002), Henry \& Fekel (2002), Henry \& Fekel (2003), Fekel et al. (2003), Mathias et al. (2004), Henry \& Fekel (2005), and Henry et al. (2005). One third of the shown $v \sin i$ values were determined in this work (dark grey). In the case of a double-lined object for which (only) one of the components is classified as a bf $\gamma$ Dor star, the companion was considered as a rejected $\gamma$ Dor star. If a double-lined object is classified as a cand/rejected $\gamma$ Dor star, both components were considered as such. The histogram of the bf $\gamma$ Dor stars shows an excess of objects within $v \sin i \in[20,60] \mathrm{km} \mathrm{s}^{-1}$ compared to the cand and rejected $\gamma$ Dor stars. These rotation rates are indeed ideal for detecting profile variations: very slow rotators require a very high spectral resolution for a detailed view of the line profile while rapid rotators suffer from line blending. There is no evidence of pulsation damping due to rapid rotation, because the relative number of stars with a high $v \sin i$ value is comparable in the 3 categories.

\section{Conclusions and future prospects}

We contributed to the observational effort to confirm cand $\gamma$ Dor stars as real members of the group of $\gamma$ Dor stars by analysing the time-series of the CORALIE spectra of 37 southern (cand) $\gamma$ Dor stars. To allow better detection of secondary components and/or profile variations, the original spectra were cross-correlated with the standard template spectrum of an F0-type star. An overview of our final results is given in Tables 1 and 3 and of our binarity and variability classification in Table 5.

At least 15 of our 37 targets turn out to be spectroscopic binaries, including 7 new ones. Our data allowed the determination of 9 orbits, of which 6 are new (Table 3): HD 34025, HD 81421, HD 214291, and HD 85964 are ellipsoidal variables, and HD 10167, HD 126516, HD 167858, HD 147787 and HD 209295 are binaries with a (cand) $\gamma$ Dor component. For HD 34025, HD 81421, and HD 85964, those phases at which $v_{\mathrm{rad}}=v_{\gamma}$ do not correspond to those phases of minimal light seen in HIPPARCOS photometry. For the remaining 6 binaries, we estimate from our data an orbital period $P_{\text {orb }} \gg 10$ days for HD 5590, HD 8393, HD 35416, and HD 110606 and $P_{\text {orb }}$ of a few days for HD 27377 and HD 111709. For the last two objects, we cannot exclude ellipsoidal variations. We also classify HD 27604, HD 26298, and HD 111829 as candidate binaries. Especially in the case of HD 26298, it is not clear if the observed variations in the CCFs are caused by binarity or not. We find a binarity rate of about $50 \%$ for our sample, which is similar to the one found by Mathias et al. (2004). If we restrict our sample to the objects that we classified as bf or cand $\gamma$ Dor stars (see column (10) in Table 1), the binary rate lowers to $\sim 40 \%$. At least 12 of our objects, i.e. $\sim 1 / 3$ of our targets, show composite spectra.

For 17 objects ( 45\%), including 3 binaries, clear crosscorrelation profile variations were observed in one of the components. We classify them as bf $\gamma$ Dor stars, except for HD 125081, which is a bf $\delta$ Sct star, and HD 111709, for which there is possible confusion with chemical peculiarity. This confirms the $\gamma$ Dor character of 10 objects (HD 14940, HD 34025, HD 40745, HD 41448, HD 112685, HD 135825, HD 149989, HD 187028, HD 216910, and HD 218225) and the $\delta$ Sct character of 1 object (HD 125081). For 8 objects, we have evidence that the intrinsic spectroscopic variations are compatible with (one of) the known photometric period(s). For 4 others, we observed no such compatibility. This group includes $\gamma$ Dor itself (HD 27290), for which our data points towards a $\delta$ Sct period instead. Due to a lack of evidence for intrinsic spectroscopic and photometric variations, we classify HD 5590, HD 7455, HD 8393, HD 22001, HD 27604, HD 33262, HD 81421, and HD 85964 as constant stars. The variability classification of our other objects remains unchanged.

The cross-correlation profiles were used to determine accurate values for the projected rotational velocity $v \sin i$ by 2 independent methods. The resulting values range from 3 to $135 \mathrm{~km} \mathrm{~s}^{-1}$. The $v \sin i$ values gathered for all the bf, cand and rejected $\gamma$ Dor stars result in decreasing histograms towards high $v \sin i$ values, except for the bf $\gamma$ Dor stars, which show more stars in the range of $[20,60] \mathrm{km} \mathrm{s}^{-1}$. 
Tidal evolution of binary systems leads to circularisation of the orbit, spin-alignment of the rotational and orbital inclination, and synchronisation of the rotation of the components with the orbital motion. For 5 double-lined binaries in our survey, i.e. HD 8393, HD 27377, HD 10167, HD 34025, and HD 214291, the $v \sin i$ values of both components are (close to) equal, which reflects their spin-alignment and synchronisation. Moreover, the orbits of HD 10167, HD 34025 and HD 214291 are circularised (Table 3 ). For the other double-lined binaries in our sample, i.e. HD 5590, HD 34025, HD 81421, HD 110606 , HD 111709, and HD 147787, the evolution towards synchronisation and/or spin-alignment is still ongoing. However, the orbits of HD 34025 and HD 81421 are already circularised (Table 3).

The binarity and variability classifications of each of our target stars were updated by the use of from 2 to 63 CORALIE spectra observed from a single site (Table 5). The low number of observations and the strong aliasing made an individual orbital or intrinsic period search difficult or impossible in several cases. Therefore, a lot of our results are based on a comparison with photometric observations of the satellite mission HIPPARcos. Clearly, additional (and ideally multi-site) spectroscopic data are needed to determine or improve orbits and to study the intrinsic variations present in spectroscopic observations in more detail. Because the exploitation of dynamical information can give additional and independent constraints on physical properties of the components, we will give priority to binaries with a bf or cand $\gamma$ Dor star in future investigations. These objects are given in italics in Table 5 .

Acknowledgements. This research was made possible thanks to financial support by the Fund for Scientific Research - Flanders (FWO), under project G.0178.02 and by the Research Council of the University of Leuven under grant GOA/2003/04. The authors did their work within the Belgian Asteroseismology Group (BAG, http://www . asteroseismology . be/). This research has made use of the SIMBAD astronomical database operated at the CDS in Strasbourg, France. We are grateful for the valuable suggestions and remarks from our referee, Dr. P. Mathias, which have improved this manuscript.

\section{References}

Aerts, C., Eyer, L., \& Kestens, E. 1998, A\&A, 337, 790

Aerts, C., De Pauw, M., \& Waelkens, C. 1992, A\&A, 266, 294

Aerts, C., Cuypers, J., De Cat, P., et al. 2004, A\&A, 415, 1079

Balona, L. A., Hearnshaw, J. B., Koen, C., et al. 1994a, MNRAS, 267, 103

Balona, L. A., Krisciunas, K., \& Cousins, A. W. J. 1994b, MNRAS, 270, 905

Balona, L. A., Bohm, T., Foing, B. H., et al. 1996, MNRAS, 281, 1315

Baranne, A., Queloz, D., Mayor, M., et al. 1996, A\&AS, 119, 373

Bertiau, F. C., \& Grobben, J. 1969, Ricerche Astronomiche Vatican Observatory, 8,1

Bouckaert, F. 2001, Frequentieanalyse voor nieuwe kandidaat- $\gamma$ Doradus sterren (Undergraduate Thesis, Katholieke Universiteit Leuven, Belgium)

De Ridder, J., Cuypers, J., De Cat, P., et al. 2004, in IAU Coll., 193, Variable Stars in the Local Group, ASP Conf. Ser., 310, 263
Duerbeck, H. W. 1997, Informational Bulletin on Variable Stars, 4513,

Dupret, M.-A., Grigahcène, A., Garrido, R., Gabriel, M., \& Scuflaire, R. 2004, A\&A, 414, L17

ESA 1997, The Hipparcos and Tycho Catalogues, ESA SP-1200 (Noordwijk: ESA)

Eyer, L. 1998, Les étoiles variables de la mission Hipparcos, Ph.D. Thesis, Geneva University, Switzerland

Eyer, L., \& Aerts, C. 2000, A\&A, 361, 201

Eyer, L., Aerts, C., van Loon, M., Bouckaert, F., \& Cuypers, J. 2002, in Observational Aspects of Pulsating B- and A Stars, ASP Conf. Ser., 256, 203

Fekel, F. C., \& Henry, G. W. 2003, AJ, 125, 2156

Fekel, F. C., Warner, P. B., \& Kaye, A. B. 2003, AJ, 125, 2196

Gray, D. F. 1992, The Observation and Analysis of Stellar Photospheres (Cambridge University press)

Grenier, S., Burnage, R., Faraggiana, R., et al. 1999, A\&AS, 135, 503

Guzik, J., \& Kaye, A. 2000, NASA STI/Recon Technical Report N, 2 , 5964

Handler, G. 1999, MNRAS, 309, L19

Handler, G. 2002, http://www . astro. univie.ac . at/ gerald/ gdorlist.html

Handler, G., Balona, L. A., Shobbrook, R. R., et al. 2002, MNRAS, 333,262

Handler, G., \& Shobbrook, R. R. 2002, MNRAS, 333, 251

Henry, G. W., \& Fekel, F. C. 2002, PASP, 114, 988

Henry, G. W., \& Fekel, F. C. 2003, AJ, 126, 3058

Henry, G. W., \& Fekel, F. C. 2005, AJ, 129, 2026

Henry, G. W., Fekel, F. C., Kaye, A. B., \& Kaul, A. 2001, AJ, 122, 3383

Henry, G. W., Fekel, F. C., \& Henry, S. M. 2005, AJ, 129, 2815

Jones, H. S. 1928, Cape. An., 10, 68

Kaye, A. B., Henry, G. W., Fekel, F. C., et al. 1999, AJ, 118, 2997

Koen, C. 2001, MNRAS, 321, 44

Koen, C., \& Eyer, L. 2002, MNRAS, 331, 45

Krisciunas, K., \& Handler, G. 1995, Informational Bulletin on Variable Stars, 4195, 1

Löffler, W. 2002, IAU Coll. 185, Radial and nonradial pulsations as probes of stellar physics, ASP Conf. Ser., 259, 508

Landolt, A. U. 1969, PASP, 81, 443

Lu, P. K., Demarque, P., van Altena, W., McAlister, H., \& Hartkopf, W. 1987, AJ, 94, 1318

Lucy, L. B., \& Sweeney, M. A. 1971, AJ, 76, 544

Mantegazza, L., Poretti, E., \& Antonello, E. 1991, Informational Bulletin on Variable Stars, 3612, 1

Martín, S., Bossi, M., \& Zerbi, F. M. 2003, A\&A, 401, 1077

Mason, B. D., Martin, C., Hartkopf, W. I., et al. 1999, AJ, 117, 1890

Mathias, P., Le Contel, J.-M., Chapellier, E., et al. 2004, A\&A, 417, 189

McAlister, H. A., Hartkopf, W. I., Hutter, D. J., Shara, M. M., \& Franz, O. G. 1987, AJ, 93, 183

Nordström, B., Stefanik, R. P., Latham, D. W., \& Andersen, J. 1997, A\&AS, 126, 21

Paunzen, E., \& Maitzen, H. M. 1998, A\&AS, 133, 1

Scargle, J. D. 1982, ApJ, 263, 835

Stellingwerf, R. F. 1978, ApJ, 224, 953

Warner, P. B., Kaye, A. B., \& Guzik, J. A. 2003, ApJ, 593, 1049

Zerbi, F. M., Garrido, R., Rodriguez, E., et al. 1997a, MNRAS, 290, 401

Zerbi, F. M., Rodríguez, E., Garrido, R., et al. 1997b, MNRAS, 292, 43

Zerbi, F. M., Rodríguez, E., Garrido, R., et al. 1999, MNRAS, 303, 275 


\section{Online Material}


P. De Cat et al.: A spectroscopic study of southern (candidate) $\gamma$ Doradus stars. I., Online Material p 2
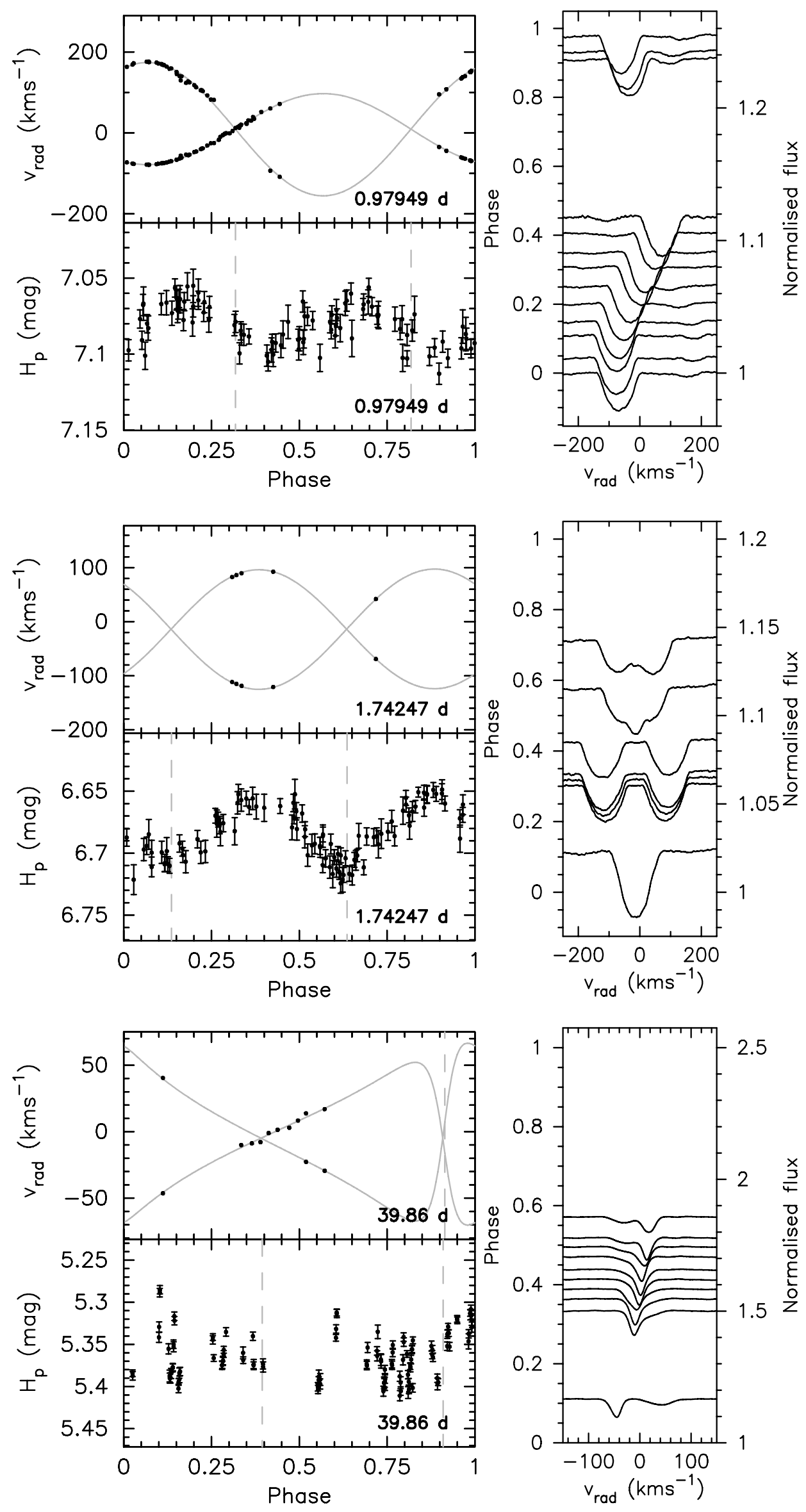

Fig. 3. Same as Fig. 1, but for HD 214291.

Fig. 6. Same as Fig. 1, but for HD 147787. The dotted line in the top left panel indicates the time of periastron passage. 
P. De Cat et al.: A spectroscopic study of southern (candidate) $\gamma$ Doradus stars. I., Online Material $p 3$
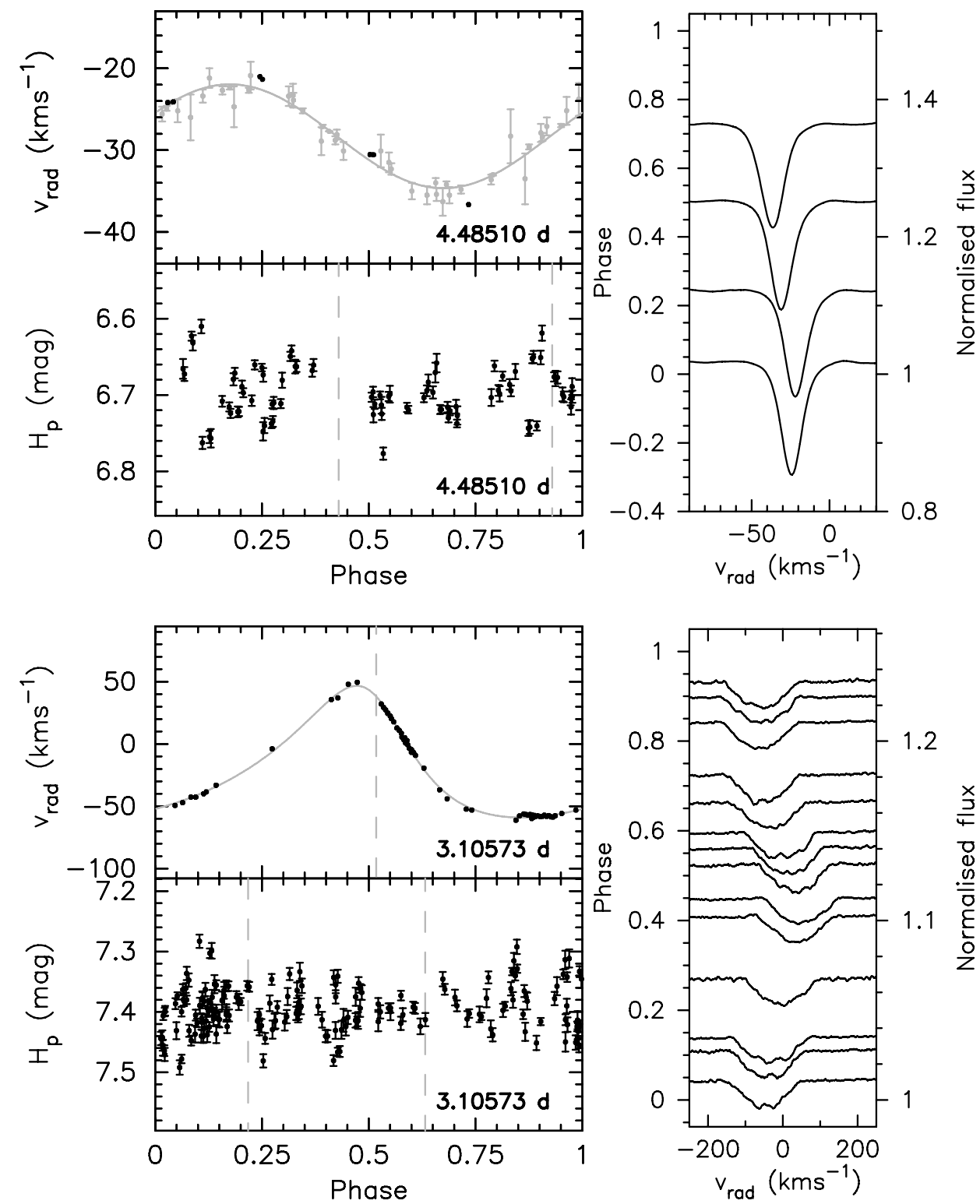

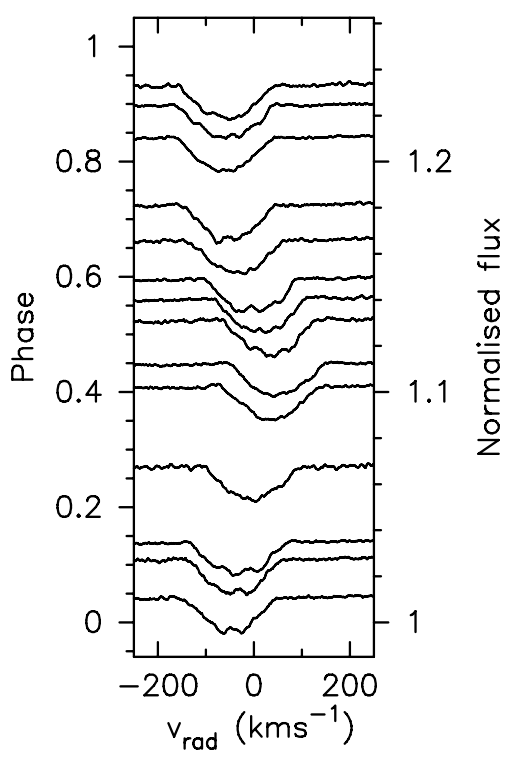

Fig. 8. Same as Fig. 1, but for HD 167858. In the top panel, the radial velocities, as determined by Fekel \& Henry (2003), are included and given in grey.
Fig. 9. Same as Fig. 1, but for HD 209295. The dotted line in the top left panel indicates the time of periastron passage. 
P. De Cat et al.: A spectroscopic study of southern (candidate) $\gamma$ Doradus stars. I., Online Material $p 4$
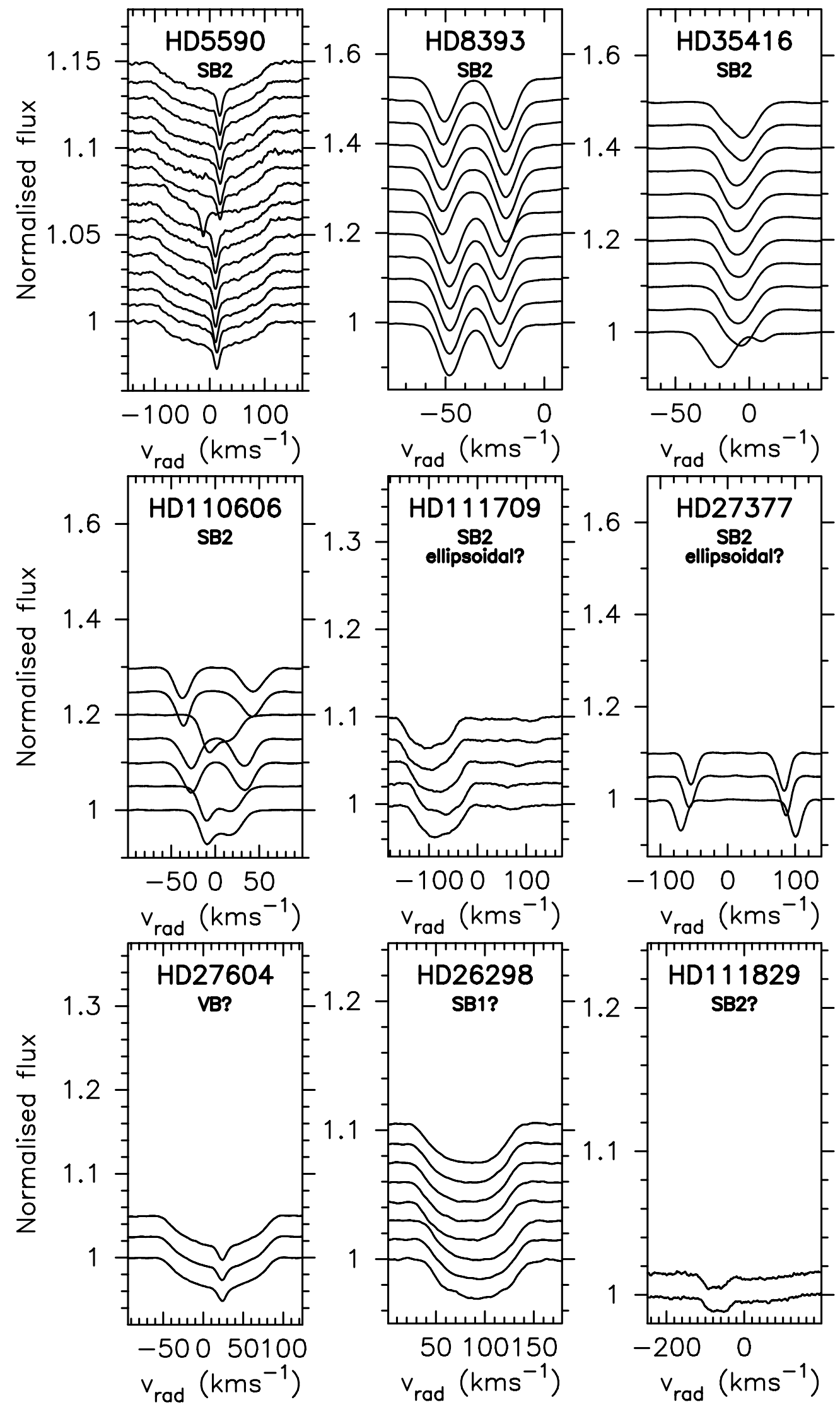

Fig. 10. The cross-correlation profiles of the observed CORALIE spectra for the (suspected) binary objects for which no orbital solution could be determined with our current set of CORALIE data. Subsequent profiles are shifted in flux for clarity. The name of the object and the orbital classification are given at the top of each panel. "SB2", "SB1", "VB", and "ellipsoidal" indicate double-lined binaries, single-lined binaries, visual binaries, and ellipsoidal variables, respectively. 
P. De Cat et al.: A spectroscopic study of southern (candidate) $\gamma$ Doradus stars. I., Online Material p 5
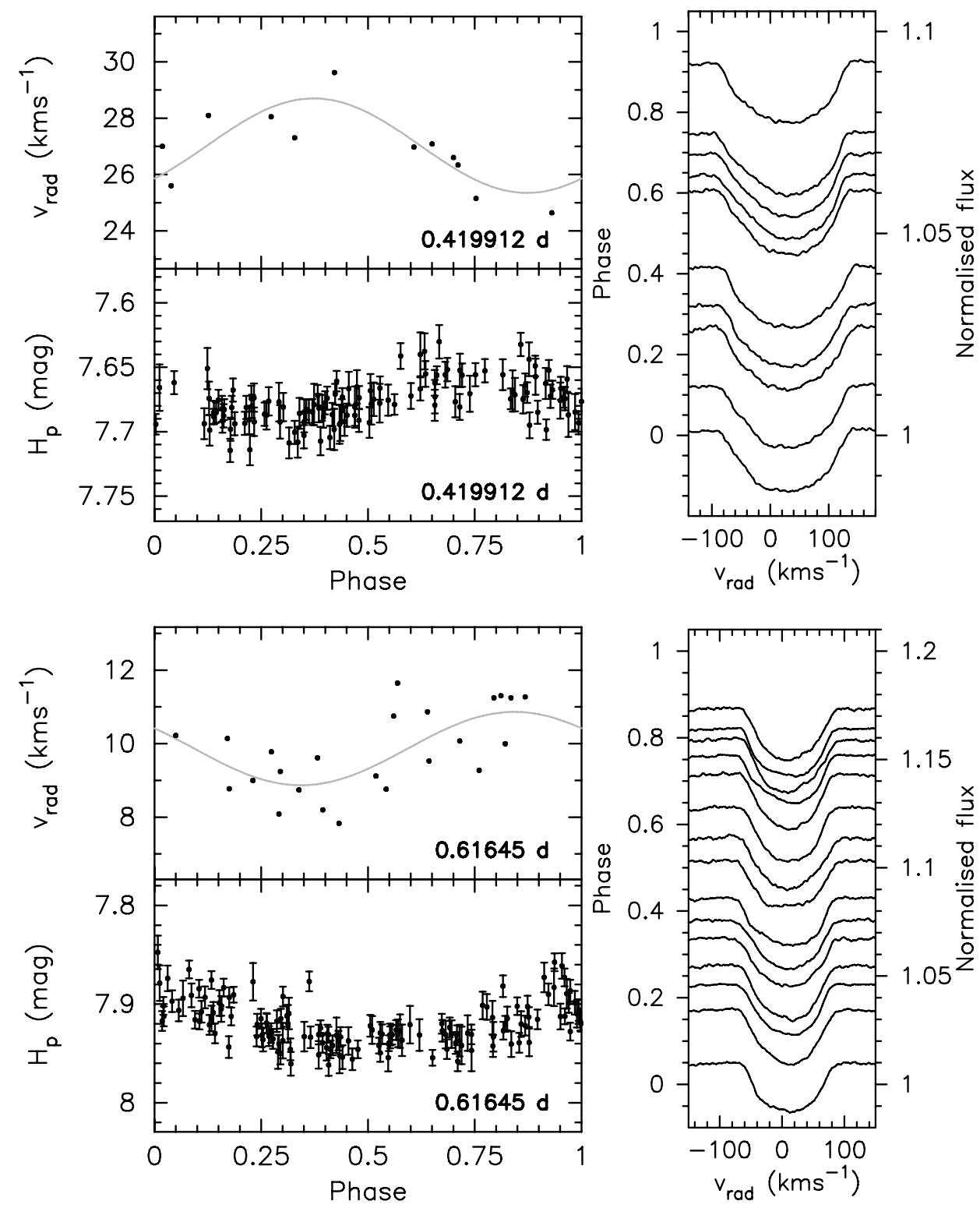

Fig. 13. Same as Fig. 12, but for HD 41448.

Fig. 14. Same as Fig. 12, but for HD 112685.
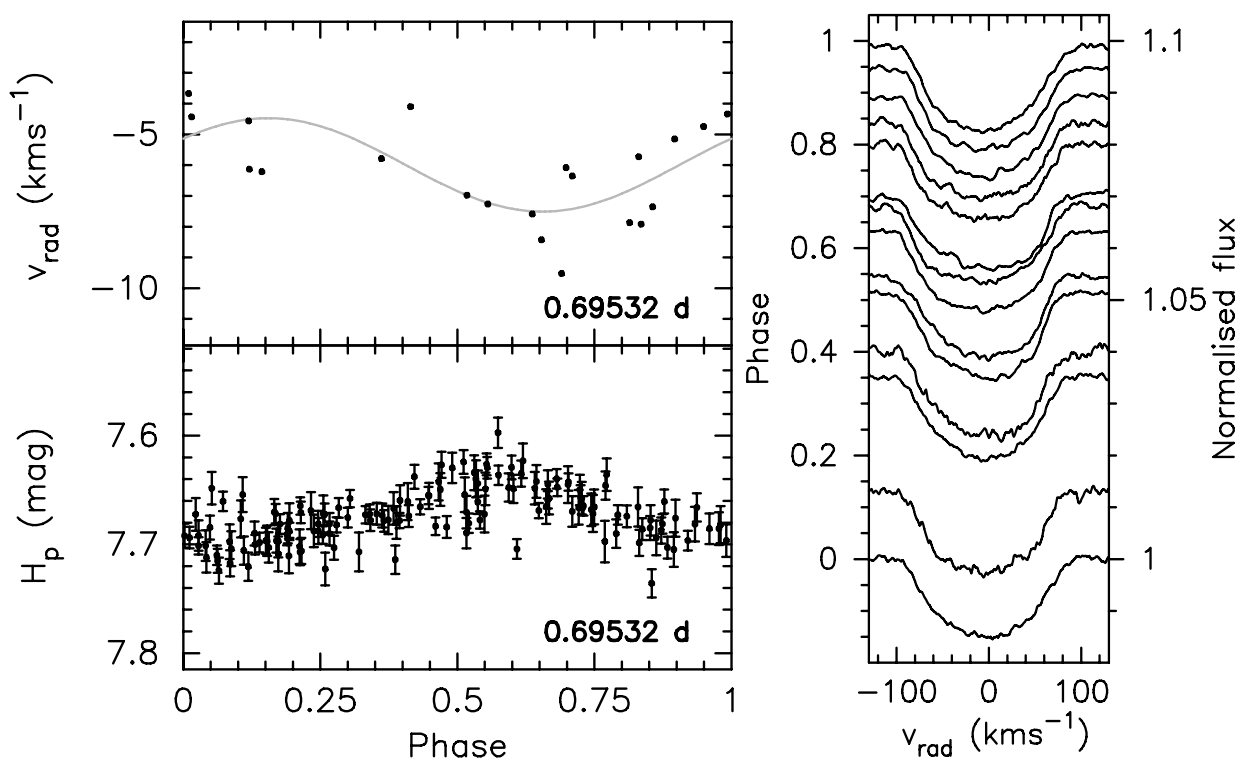

Fig. 15. Same as Fig. 12, but for HD 187028. 
P. De Cat et al.: A spectroscopic study of southern (candidate) $\gamma$ Doradus stars. I., Online Material p 6
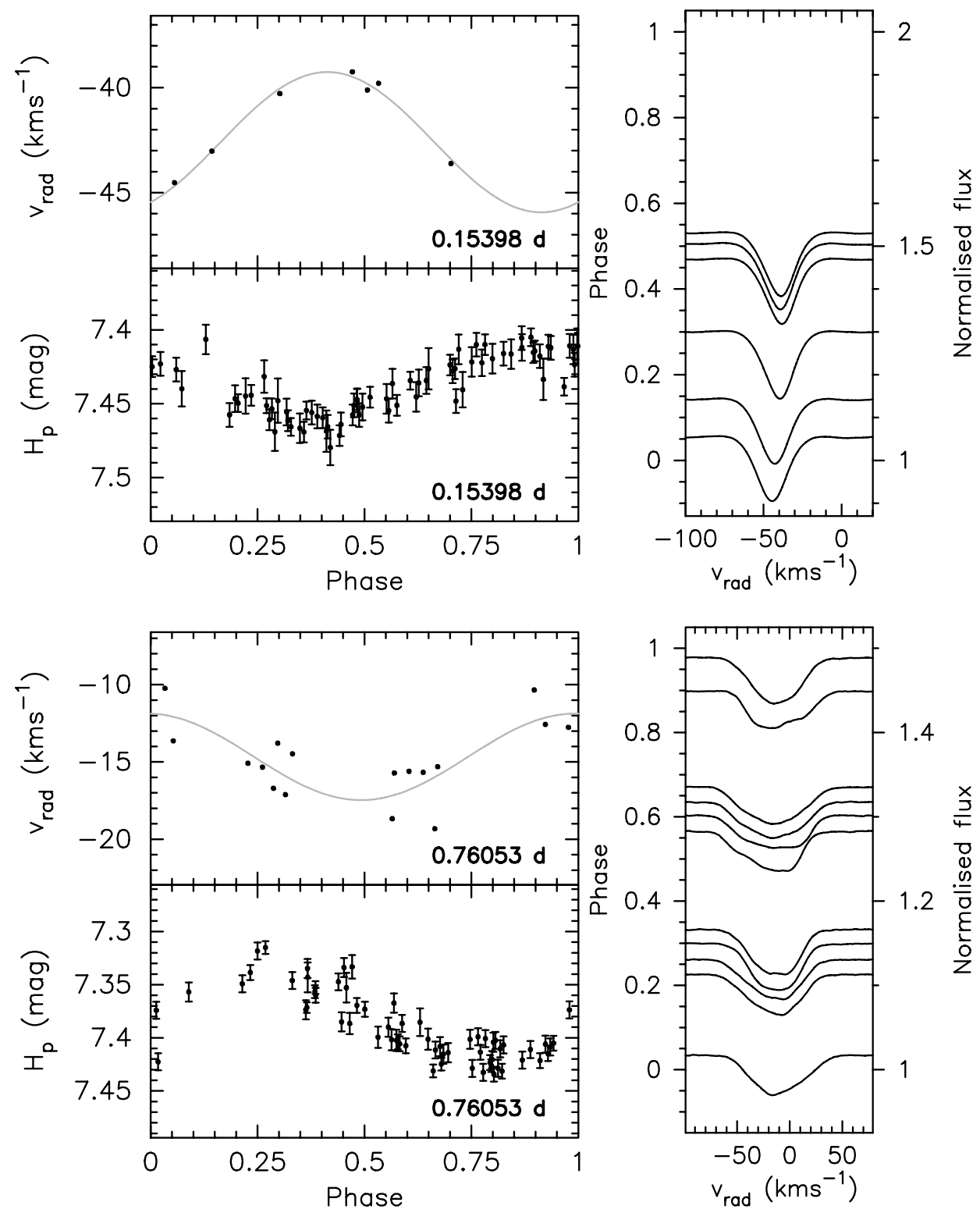

Fig. 16. Same as Fig. 12, but for HD 125081.

Fig. 17. Same as Fig. 12, but for HD 135825.
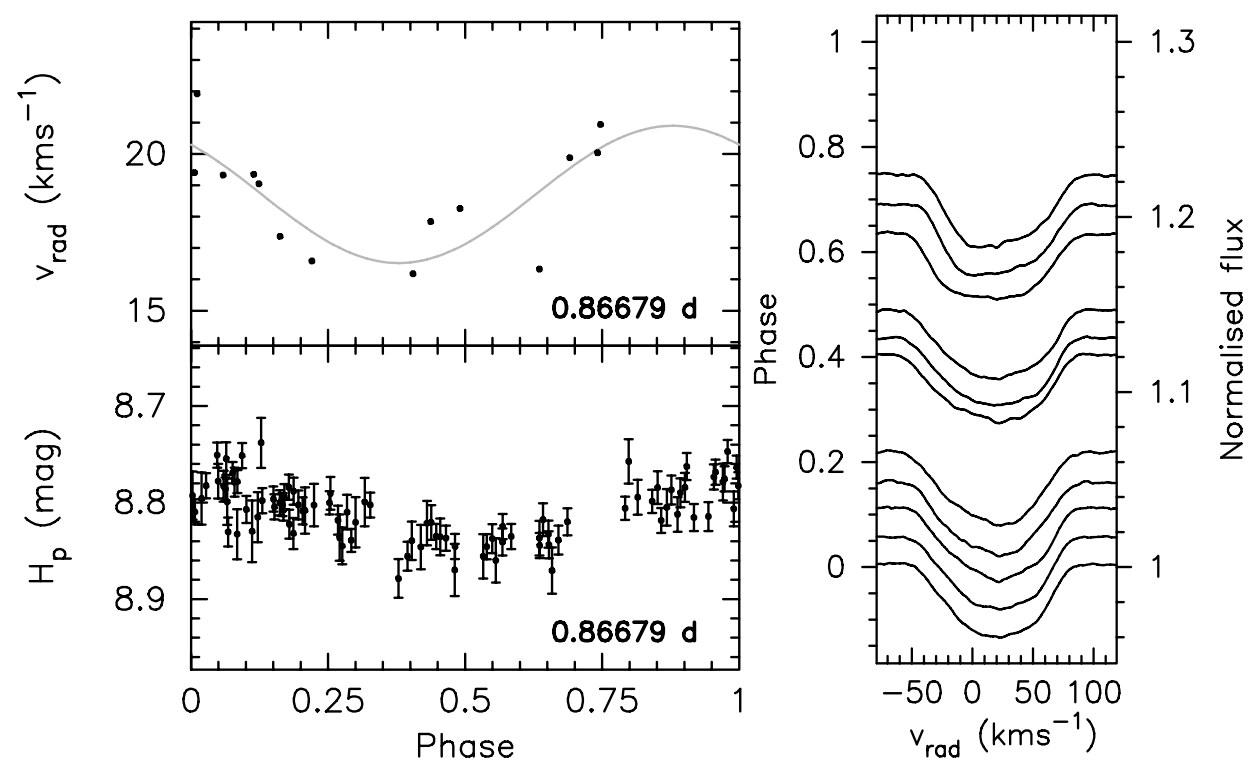

Fig. 18. Same as Fig. 12, but for HD 218225 . 
P. De Cat et al.: A spectroscopic study of southern (candidate) $\gamma$ Doradus stars. I., Online Material $p 7$
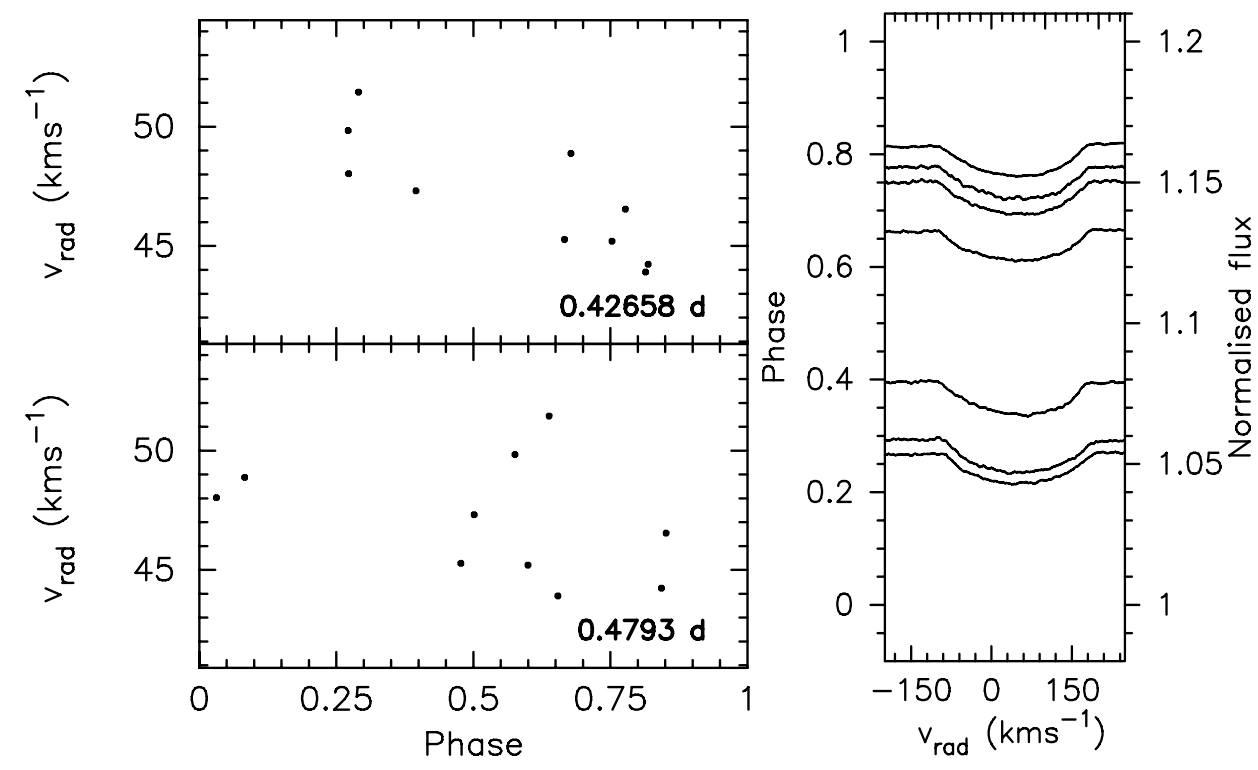

Fig. 21. Phase diagrams of the radial velocity $v_{\text {rad }}$ with two known HIPPARcos periods of HD 149989 (left). The period values are given in the bottom right corner. The reference epoch is HJD 2450000. In the right panel, a selection of observed cross-correlation profiles are shown as a function of the main HIPPARCOS period.
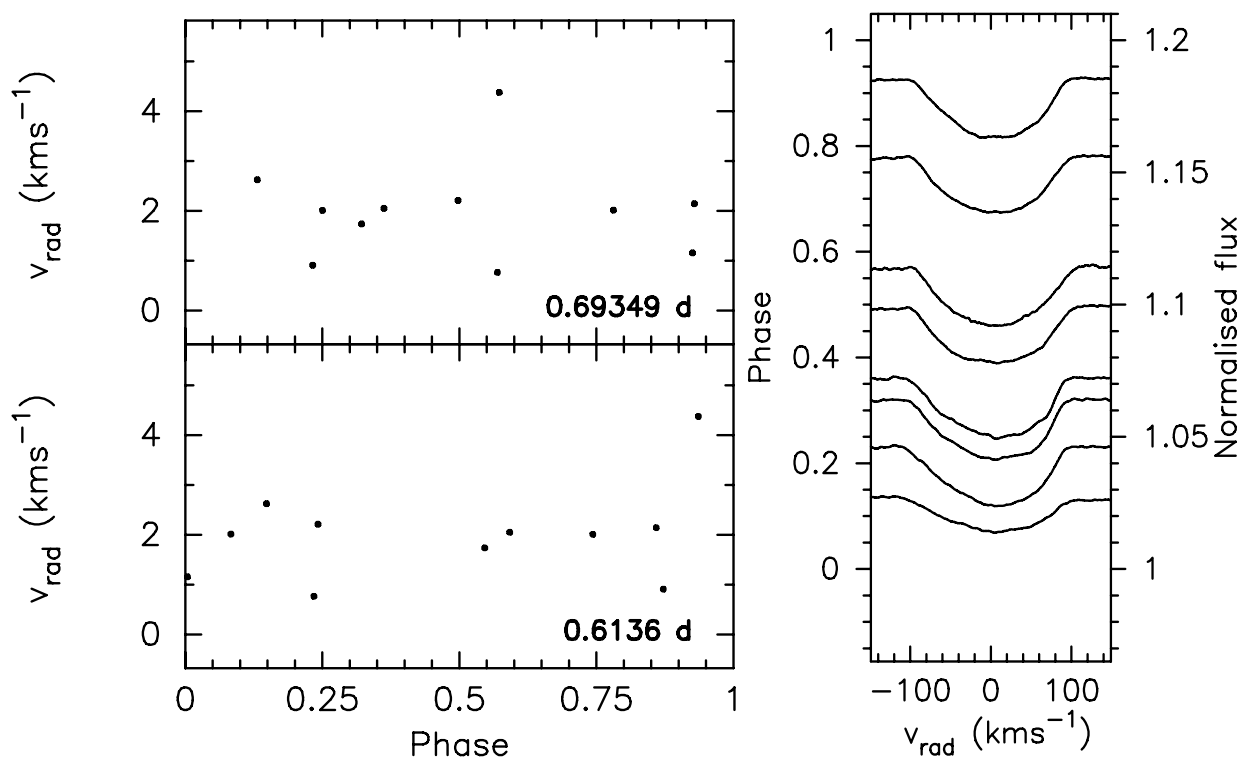

Fig. 22. Same as Fig. 21, but for HD 216910. 
P. De Cat et al.: A spectroscopic study of southern (candidate) $\gamma$ Doradus stars. I., Online Material $p 8$

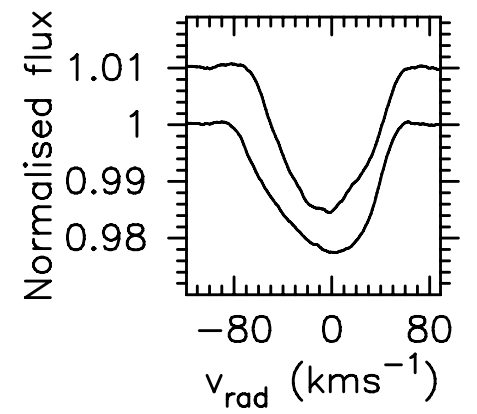

Fig. 23. The cross-correlation profiles of the observed CORALIE spectra for HD 65526. Subsequent profiles are shifted in flux for clarity. 
P. De Cat et al.: A spectroscopic study of southern (candidate) $\gamma$ Doradus stars. I., Online Material p 9
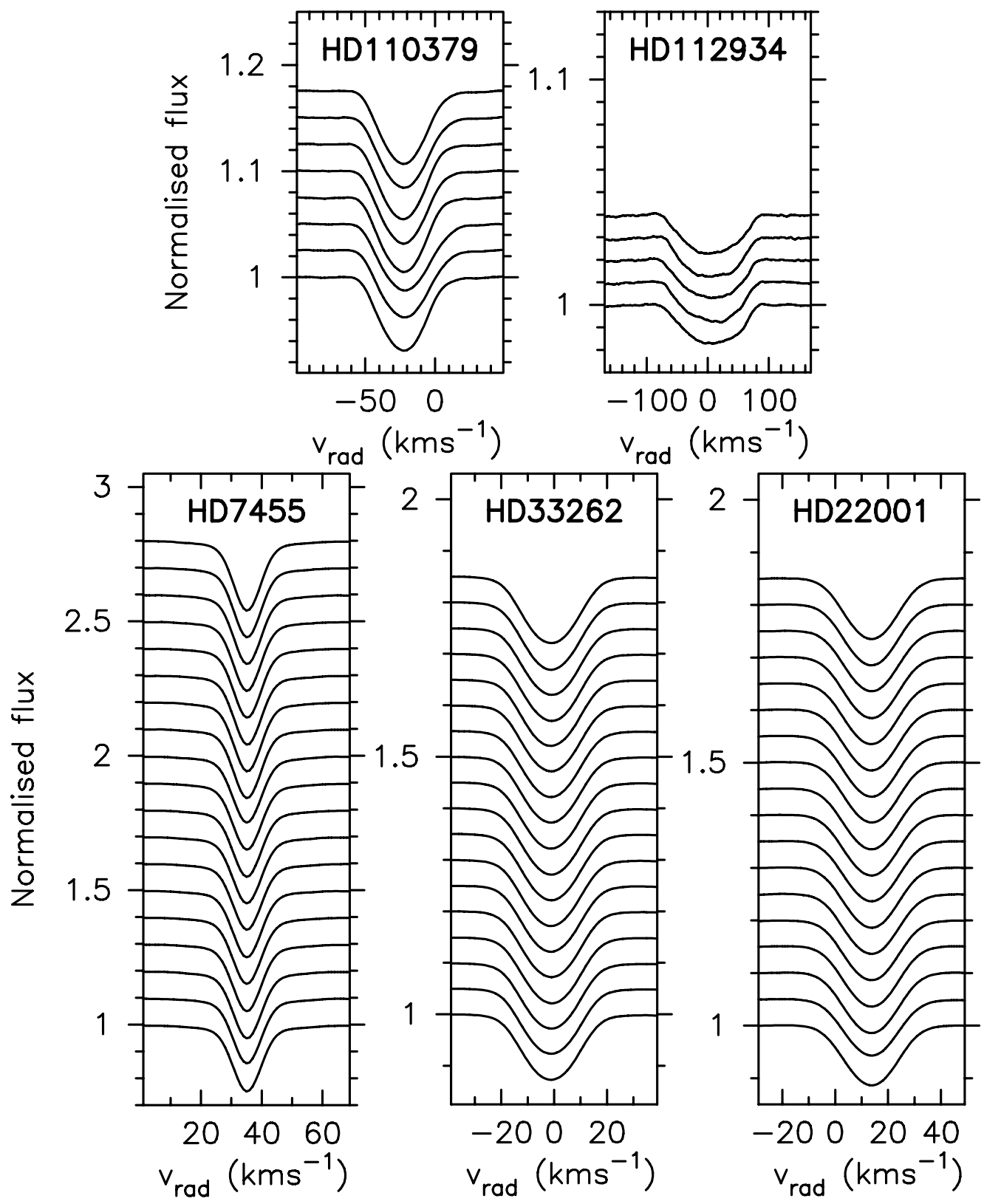

Fig. 24. The cross-correlation profiles of the observed CORALIE spectra for the objects for which no clear correlation profile variations have been detected in our current set of CORALIE data. Subsequent profiles are shifted in flux for clarity. The HD number of the object is given at the top of each panel. 